\title{
Procesos de fabricación de metales celulares. Parte Il: Vía sólida, deposición de metales, otros procesos ${ }^{(\cdot)}$
}

\author{
P. Fernández*, L.J. Cruz* y J. Coleto**
}

\begin{abstract}
Resumen
En la primera parte de esta revisión se realizó la descripción de los procesos empleados por vía líquida para la fabricación de metales celulares. En esta segunda parte, se describen los procesos correspondientes a la vía sólida y la deposición de metales. De igual manera, se revisan los diferentes métodos, en cada caso, haciendo una breve descripción de los principales parámetros involucrados y las ventajas y desventajas en cada uno de ellos.
\end{abstract}

Palabras clave Metales celulares; Espumas metálicas; Esponjas metálicas; Pulvimetalurgia; Deposición de metales.

\section{Manufacturing processes of cellular metals. Part II. Solid route, metals deposition, other processes}

\begin{abstract}
At the first part of this paper review a description about cellular metal processes by liquid route, was made. In this second part, solid processes and metals deposition are described. In similar way, the different kind of processes in each case are reviewed; making a short description about the main parameters involved and the advantages and drawbacks in each of them.
\end{abstract}

Keywords

\section{INTRODUCCIÓN}

Los procesos de producción de metales celulares (MC) por ruta sólida han avanzado últimamente, en lo que a innovación y optimización se refiere. Dentro de los métodos existentes para la fabricación de metales celulares, los procesos pulvimetalúrgicos se han ido posicionando como los de mayor investigación y evolución, aunque no son propiamente los más económicos a la hora de implementarse. Así mismo, los metales celulares obtenidos por la ruta de deposición gaseosa de metales han evolucionado sustancialmente a través de los años constituyéndose, actualmente, como la ruta preferida para conseguir esponjas metálicas para aplicaciones netamente funcionales. Además, se describen otras rutas menos exploradas pero que dan cuenta del interés generado por la producción de metales celulares a nivel mundial.

\section{FABRICACIÓN DE MC POR VÍA SÓLIDA}

\subsection{Espumación de preformas produci- das por pulvimetalurgia (PM)}

El Fraunhofer-Institute for Manufacturing and Advance Materials (IFAM, Bremen, Alemania) desarrolló este método para la producción de espumas por PM, denominado Foaminal ${ }^{[1]}$. Ahora, son varias las empresas e institutos que producen espumas a través de este proceso: Mepura (Alulight) y Neuman Aluminium, son dos de ellas ${ }^{[2-9]}$. Cabe anotar que la espumación de la preforma obtenida se puede hacer en molde abierto o con formas complejas 3D, o bien formando estructuras tipo sándwich donde una piel metálica (acero o aluminio) o cerámica (alúmina) cubre un núcleo de espuma metálica de aluminio ${ }^{[1}$ y 10-13]. El aluminio ha sido utilizado, ampliamente, como material de relleno en

\footnotetext{
(•) Trabajo recibido el día 12 de febrero de 2008 y aceptado en su forma final el día 7 de julio de 2008.

* Grupo de Investigación sobre Nuevos Materiales, Universidad Pontificia Bolivariana, Medellín, Colombia patricia.fernandez@upb.edu.co, luis.cruz@upb.edu.co.

**Fundación Inasmet-Tecnalia, San Sebastián, España, javier.coleto@inasmet.es,
} 
estructuras tipo sándwich y perfiles, mostrando un comportamiento sobresaliente en varias propiedades mecánicas, tales como la capacidad de absorción de energía de deformación, la tenacidad, la resistencia a la flexión y la resistencia mecánica ${ }^{[14]}$.

En un primer paso, los polvos metálicos (usualmente aleaciones de aluminio) y el agente espumante (comúnmente $\mathrm{TiH}_{2}$ ) y otros aditivos (partículas cerámicas que actúan como estabilizadoras de la espuma) se mezclan de manera homogénea, lo cual permite conseguir componentes de calidad con una distribución de tamaño de poro controlada. En un segundo paso, para la obtención de las preformas espumables, la mezcla puede ser compactada por presión uniaxial, laminación, compresión isostática en frío (método más prometedor para la obtención de grandes componentes semiacabados de geometría variada o extrusión ${ }^{[15-18]}$; esta última, considerada la vía más económica y más adaptable a nivel industrial. Una práctica común consiste en someter inicialmente los polvos a un proceso de compactación en frío, donde se obtiene una preforma compactada que, posteriormente, se somete a extrusión, garantizando, de esta forma, menor contaminación y mejor manipulación del compacto ${ }^{[19]}$. En la unión por laminación, las láminas obtenidas pueden ser espumadas tal cual o se pueden unir con otras láminas de aluminio o acero (por una o dos caras) que, después de ser espumadas, originan estructuras tipo sándwich de altas prestaciones. Sin embargo, debe tenerse en cuenta que la laminación en caliente puede presentar mayores dificultades ${ }^{[20]}$. Después de la compactación se realiza la espumación, colocando el material precursor en un molde, donde se calienta a una temperatura cercana a la de fusión del metal de la matriz a fin de generar la descomposición del agente espumante y, así, la liberación del gas que crea la estructura porosa ${ }^{[21-23]}$. Una vez alcanzada la espumación se procede a un enfriamiento rápido. La figura 1 muestra un esquema del proceso.

De acuerdo a los datos dados a conocer por diferentes autores, las espumas obtenidas mediante esta vía suelen tener porosidades comprendidas entre 50 $92 \%$ [24], con tamaños de poro promedio entre $0,5 \mathrm{y}$ $8 \mathrm{~mm}{ }^{[25]}$ y con densidades típicas en un intervalo de $0,4-0,8 \mathrm{~g} / \mathrm{cm}^{3}[26]$. La densidad relativa en el caso del aluminio y del acero suele ser de alrededor del $20 \mathrm{y}$ $40 \%$, respectivamente ${ }^{[27]}$. Otro camino posible consiste en someter la preforma compactada a un calentamiento seguido de un procesamiento por thixocasting (tixoformado), método que se desarrolla en estado semisólido y el cual es un híbrido que combina aspectos de fundición y de pulvimetalurgía ${ }^{[28-33]}$. Para obtener componentes finales espumados o con núcleos en espuma, se ha puesto a prueba la combinación entre la vía de PM con la técnica de moldeo por inyección, donde la espuma, aún en estado semisólido, se inyecta de manera controlada en un molde

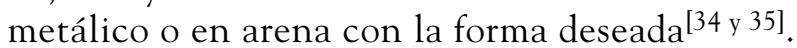
Aunque las aleaciones de aluminio, tales como la 7075, 6061, AlSi7Mg (A356), AlSi12, AlSi7, AlMgSi0.6, AlSi6, AlSi6Cu4 son las más utilizadas para espumar por este método ${ }^{[22}$ y 36-38], seleccionando los parámetros de proceso y el agente espumante adecuados ${ }^{[26]}$; también, es posible espumar por PM metales, tales como el estaño, zinc ${ }^{[39]}$, bronce, plomo, oro, $\operatorname{aceros}^{[27 \text { y 40] }}$ titanio $^{[41 \text { y } 42]}$.

\subsubsection{Influencia de los parámetros del proceso}

En la preparación del precursor, algunos de los parámetros de mayor relevancia son el contenido de agente espumante, la presión y la temperatura de

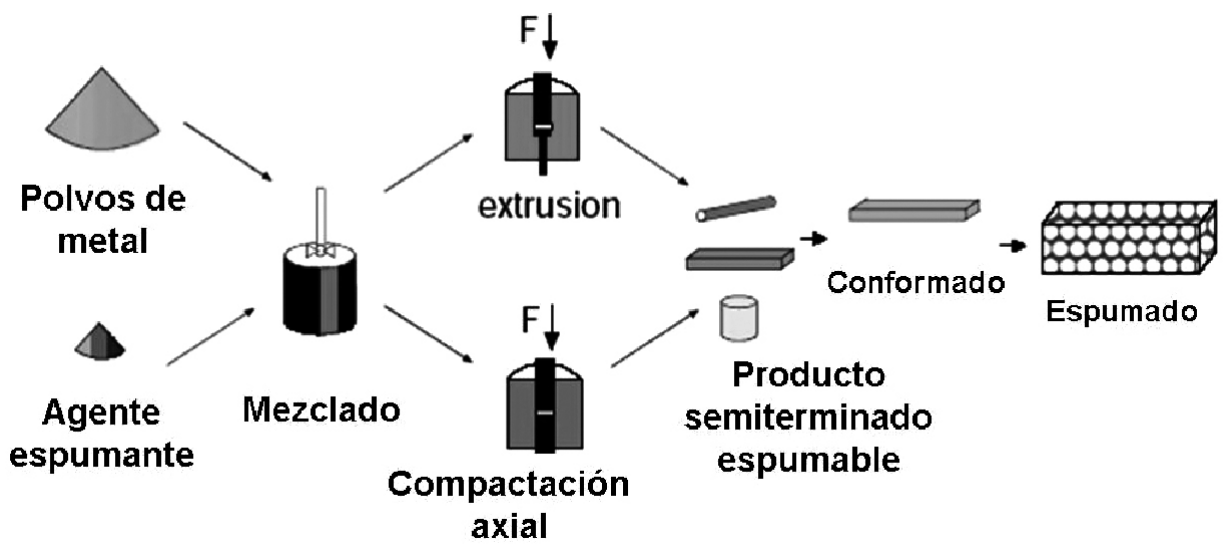

Figura 1. Proceso de espumado por PM [21].

Figure 1. PM foaming process ${ }^{[21]}$. 
compactación $^{[43]}$. Adicionalmente, la densidad del producto se puede controlar por medio de parámetros como las velocidades de calentamiento, el equipo utilizado para la compactación, la forma y el material del molde, la temperatura de sinterización o el tipo de horno, entre otros. Además de los parámetros que se explicarán a continuación, también, influyen de manera decisiva las propiedades de los polvos empleados, tales como la forma, el tamaño y la distribución de las partículas, la homogeneidad de la mez-

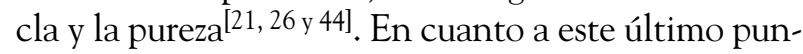
to, se ha observado que la presencia de óxidos, tanto en la mezcla de polvos como propios del aluminio, tiene un efecto significativo sobre la expansión y estabilidad de las espumas elaboradas por PM [45 y 46]. Cabe anotar que, en algunos casos, se han aplicado técnicas estadísticas enfocadas a la optimización de los parámetros de proceso y su influencia en las propiedades de las espumas obtenidas ${ }^{[6]}$.

\subsubsection{Efecto de la compactación}

Se ha demostrado que las condiciones de compactación tienen un efecto importante sobre la cinética del proceso de espumación, siendo posible obtener una descomposición considerablemente mayor a la temperatura de fusión del metal cuando se ha realizado una compactación significativa ${ }^{[47]}$. Es así, cómo parámetros tales como la tecnología de compactación utilizada, el grado de deformación, el tiempo, la temperatura y la presión de compactación tienen una fuerte influencia en la etapa de espumación ${ }^{[18,21,26 \text { y 48]. }}$ Asavavisithchai y Kennedy estudiaron la dependencia de las características microestructurales presentadas por espumas obtenidas con precursores de polvos compactados mediante cuatro métodos diferentes de compactación, demostrando que se presentan varios niveles de cizalladura y rompimiento de óxidos durante la consolidación que generan reacciones de las partículas que rodean el $\mathrm{TiH}_{2}$ y evidenciando así mismo signos de disolución de titanio en la matriz ${ }^{[49]}$.

\subsubsection{Efecto de las partículas cerámicas}

Uno de los efectos más importantes sobre la estabilidad de la espuma y sus propiedades mecánicas está relacionado con las partículas cerámicas (usualmente $\mathrm{SiC}$, $\mathrm{Al}_{2} \mathrm{O}_{3}, \mathrm{TiB}_{2}$ ) mezcladas con los polvos del metal y del espumante, cuya función como reforzante y estabilizador en la obtención de espumas depende fuertemente del porcentaje utilizado, del tamaño y del tipo de partículas ${ }^{[50-52]}$. El papel que juegan las partículas ce- rámicas en la estabilización de la espuma del metal líquido ha sido analizado desde el punto de vista de las fuerzas interfaciales y de la viscosidad [53], así como su influencia en el espesor de la pared de la celda y el tiempo de expansión de la espuma.

\subsubsection{Parámetros relacionados con la espumación}

Banhart et al. indagaron acerca de la influencia de la cantidad de agente espumante sobre la densidad del metal espumado ${ }^{[54]}$, mientras que Centeno et al. estudiaron las interacciones gas-líquido durante el espumado de los polvos compactos ${ }^{[55]}$. El agente espumante, en el caso de las aleaciones de aluminio, suele usarse en cantidades menores al $1 \%$ en peso (usualmente $\mathrm{TiH}_{2}$ ) [24 y 36]. Se debe tener en cuenta que, aunque los polvos de $\mathrm{TiH}_{2}$ comienzan su descomposición a $\sim 380^{\circ} \mathrm{C}$ y continúa por encima de los $570^{\circ} \mathrm{C}$, su comportamiento puede variar cuando se encuentra mezclado y compactado con los polvos de la aleación ${ }^{[56]}$; además, hay que anotar que su liberación es mayor y más rápida a temperaturas más altas ${ }^{[57]}$. Matijasevic et al. mostraron que un pre-tratamiento de oxidación superficial a temperaturas entre $440-520^{\circ} \mathrm{C}$, realizado sobre el espumante antes de la compactación, tiene efectos benéficos sobre la

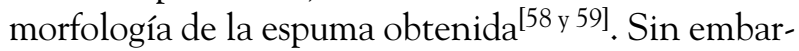
go, Kennedy et al. afirman que un tratamiento térmico de mayor exposición sobre el $\mathrm{TiH}_{2}$ puede ser perjudicial porque reduciría la cantidad de gas disponible para la espumación ${ }^{[60 \text { y 61] }}$.

Estudios realizados por diferentes investigadores han demostrado que la espumación depende, básicamente, de la temperatura utilizada durante la compactación de los polvos, la temperatura de espumado ${ }^{[62}$ y 63] , la velocidad de calentamiento, el tiempo

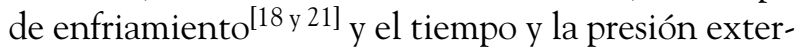
na sobre la espuma ${ }^{[37]}$. En cuanto a la cinética de expansión de las espumas de aluminio, se ha encontrado que ésta es influenciada por la tensión superficial de la aleación ${ }^{[18]}$, la composición de la aleación ${ }^{[38}$ y ${ }^{44]}$ y la atmósfera de trabajo ${ }^{[64]}$. Por otra parte, a fin de tener un mayor control sobre el tamaño de los poros, es importante buscar un equilibrio entre la presión externa, la cual soporta la tensión superficial de la aleación, y la presión de gas interna de los poros en la espuma. Con respecto a este tema, Banhart realizó un estudio muy completo con referencia al fenómeno de estabilización de la espuma ${ }^{[65-68]}$, del que concluyó que la espumación se desarrolla a través de las tres etapas esquematizadas en la figura 2.

Es evidente que el proceso de espumación es bastante sensible y fuertemente gobernado por el efecto 


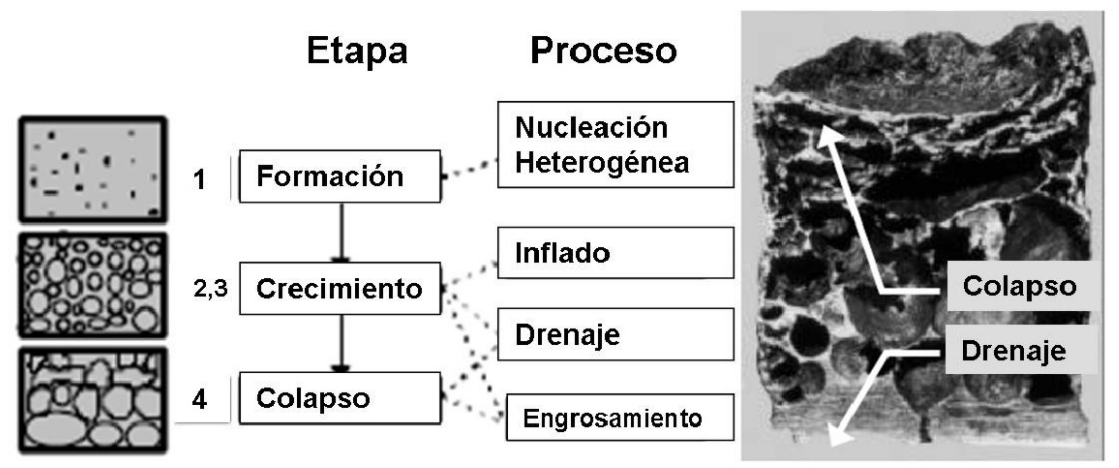

Figura 2. Etapas de la espumación, drenaje y colapso parcial por sobrecalentamiento [68].

Figure 2. Foaming, drainage and partial collapse phases caused by overheating [68].

de las diferentes temperaturas: la de compresión en caliente, la de espumado, la velocidad de calentamiento, la del horno utilizado para el espumado y la

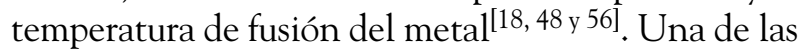
innovaciones más recientes en el espumado de preformas obtenidas por PM es el calentamiento por láser de $\mathrm{CO}_{2} / \mathrm{Nd}$-YAG, éste permite generar una expansión localizada y unidireccional de la espuma metálica, predominantemente, en la dirección del rayo láser. De esta manera, es posible producir espumas de aluminio con densidad relativa entre 0,33-0,39 [69].

Otro de los aspectos de mayor relevancia durante el espumado lo constituyen los gradientes de temperatura, por lo que se considera clave tratar de coordinar la temperatura de descomposición del agente espumante con la temperatura de fusión del metal de la aleación matriz ${ }^{[62}$ y 63], en cuyo caso se considera que las temperaturas típicas del proceso Foaminal para las aleaciones de aluminio están comprendidas entre 590 y $750^{\circ} \mathrm{C}$ y para las aleaciones de zinc, entre 420 y $600^{\circ} \mathrm{C}[5]$.

Existen varios estudios de caracterización del proceso de espumación realizados mediante el uso de avanzadas técnicas, tales como monitoreo de imágenes a diferentes tiempos de espumación ${ }^{[70]}$, tomografía de RX ${ }^{[71-73]}$, microtomografia de alta resolución ${ }^{[74-}$ ${ }^{76]}$, por microgravedad ${ }^{[77-81]}$ radioscopia de $\mathrm{RX}^{[82]}$, barrido de neutrones de ángulo pequeño ${ }^{[83-85]}$, XRD ${ }^{[86]}$, entre otros. Brunke y Odenbach, por su parte, realizaron experimentos encaminados a demostrar la influencia de las fuerzas de Lorentz y las fuerzas rotacionales en el comportamiento de las espumas líquidas ${ }^{[87]}$. Aún así, continúan existiendo muchos vacíos por cubrir en cuanto al comportamiento de las espumas durante la etapa de espumación.

De otro lado, varios estudios han sido enfocados hacia la caracterización y optimización de agentes espumantes con miras al mejoramiento de la calidad de las espumas obtenidas. Entre los agentes espuman- tes de mayor uso en el proceso de PM están el $\mathrm{TiH}_{2}$, $\mathrm{ZrH}_{2}, \mathrm{CaH}_{2}, \mathrm{MgH}_{2}$ y el $\mathrm{HfH}_{2}$, empleados para el espumado de aleaciones base zinc y base aluminio, debido a que sus temperaturas de descomposición caen dentro del intervalo de temperaturas donde dichas aleaciones alcanzan el estado líquido ${ }^{[88 \text { y }}{ }^{89]}$. Debido al alto punto de fusión de las aleaciones de hierro, la PM es el método más utilizado para su espumado ${ }^{[00}$ y 91], en cuyo caso, se emplean como agentes espumantes los siguientes: $\mathrm{MgO}_{3}{ }^{[91]}, \mathrm{Cr}_{\mathrm{x}} \mathrm{N}^{\text {[40 y }}$ 92], $\mathrm{MnN}$, $\mathrm{MoN}, \mathrm{CaCO}_{3}, \mathrm{BaCO}_{3}$ y $\mathrm{SrCO}_{3}[26,40$ y 93$]$, siendo este último el que mejores resultados ha mostrado para producir espumas con una densidad aproximada de $3 \mathrm{~g} / \mathrm{cm}^{3}{ }^{340]}$. Así mismo, el plomo ha sido espumado con $\left(\mathrm{PbCO}_{3}\right)_{2} \cdot \mathrm{Pb}(\mathrm{OH})_{2}{ }^{[26,70,77 \text { y } 81]}$ y se ha utilizado satisfactoriamente el $\mathrm{B}_{4} \mathrm{C}$ como agente espumante para la obtención de espumas de compuestos de titanio ${ }^{[94]}$. El $\mathrm{CaCO}_{3}$ también ha sido utilizado satisfactoriamente para la estabilización in-situ del fundido durante el espumado de aluminio, produciendo espumas con una densidad más baja y tamaños de poro más pequeños. En algunos casos, se han utilizado mezclas de dos agentes espumantes a fin de obtener intervalos de temperatura más amplios durante la liberación del hidrógeno, lo que conduce a su eficiente utilización, una máxima expansión y un mínimo colapso de la espuma de zinc ${ }^{[39]}$.

El uso de precursores elaborados con polvos del metal y agente espumante es una técnica alternativa del proceso por pulvimetalurgia. Esta consiste en obtener, inicialmente, los precursores mediante diferentes métodos: corte, perforado o estampado, para luego ser espumados en conjunto ${ }^{[95]}$. Otra técnica consiste en obtener el compacto de la mezcla de polvos de metal y polvos del agente espumante y, previamente a la sinterización se realiza el maquinado, directamente del compacto, a la forma final deseada, luego, se procede a la remoción de los aditivos y la sinterización de la espuma ${ }^{[96]}$. El IFAM ha desarrollado una 
novedosa espuma denominada APM por su sigla en inglés de Advance Pore Morphology, la cual se caracteriza por tener dos tipos de porosidad en su conjunto: una interna de tipo cerrado que es propia de cada elemento que compone la espuma y la porosidad abierta del material ${ }^{[7]}$. De otro lado, en la ruta por PM se ha intentado el uso de materiales reciclados por dos vías: por un lado, reemplazando los polvos metálicos por virutas metálicas generadas en procesos de cor$\mathrm{te}^{[33]} \mathrm{y}$, por el otro, mediante la sustitución del agente espumante por materiales de desecho ricos en carbonatos de calcio y magnesio provenientes de actividades mineras ${ }^{[97]}$. Para finalizar, a continuación, se indican las ventajas y desventajas de este proceso:

\subsubsection{Ventajas}

- Tecnología madura para producción a gran escala.

- Amplio rango de densidades.

- Estructuras sándwich con unión metálica o cerámica.

- Proceso relativamente sencillo usando $\mathrm{TiH}_{2} \mathrm{O}$ $\mathrm{ZrH}_{2}$ como agente espumante.

- Gran abanico de formas. Espumación tras conformado.

- Posibilidad de obtener piezas huecas con núcleo de espuma.

\subsubsection{Desventajas}

- Proceso más costoso que la ruta líquida.

- Limitación de tamaño.

- Dificultades en el control de tamaño de poros.

- Hidruros metálicos liberan $\mathrm{H}_{2}$.

- Parámetros de proceso no optimizados y los efectos inevitables de la gravedad causan efectos adversos sobre el proceso de espumado.

- Difícil lograr la reproducibilidad de las espumas.

\subsection{Sinterización de mezcla de polvos metálicos - material removible (space holder)}

Este método ha sido desarrollado en la Universidad de Liverpool, donde el investigador Zhao et al. han trabajado bastante en este proceso alternativo denominado SDP (Sintering Disolution Process) ${ }^{[98]}$. Este, consiste en mezclar polvos en una relación determinada, según la porosidad deseada, de un metal y de un material que permita su remoción al final del proceso. Zhao ha utilizado ampliamente mezclas de pol- vos de aluminio con $\mathrm{NaCl}$, donde el tamaño de los polvos de aluminio no es crítico ya que el tamaño de poro de las celdas es determinado por el tamaño de los granos de sal o de cualquier otro material utilizado en su lugar, el cual es comúnmente llamado powder space holder (PSH) ${ }^{[99]}$. Una vez mezclados los polvos del metal con los de $\mathrm{NaCl}$, se compactan, obteniéndose una preforma que posteriormente pasa a ser sinterizada a una temperatura por debajo de la temperatura de fusión del $\mathrm{NaCl}$ y cercana o igual a la temperatura de fusión del metal $\left(\mathrm{T}_{\mathrm{f} \mathrm{NaCl}}=801^{\circ} \mathrm{C}\right.$, $\mathrm{T}_{\text {sinterización }}=610$ a $680^{\circ} \mathrm{C}$ ). Luego, se procede a la disolución del $\mathrm{NaCl}$ en agua para obtener una esponja de aluminio. La velocidad de disolución del $\mathrm{NaCl}$ es aproximadamente proporcional a la fracción en volumen de $\mathrm{NaCl}$ en el compacto inicial de $\mathrm{Al} / \mathrm{NaCl}$ e inversamente proporcional a la raíz cuadrada del tiempo de disolución ${ }^{[100]}$. Mediante SDP es posible obtener componentes de una porosidad entre un 50 y un $85 \%$, con tamaños de celda que se encuentran entre 100 y $5.000 \mu \mathrm{m}^{[101]}$. En la figura 3 se puede ver el esquema del proceso.

Uno de los estudios de Zhao ${ }^{[101]}$ muestra cómo los polvos de magnesio pueden ser usados para mejorar la aglomeración de partículas de aluminio durante la sinterización, reduciendo en cierta medida el efecto de la presencia de óxidos de aluminio. Sin embargo, se han encontrado trabajos que indican que las esponjas de aluminio obtenidas por SDP poseen bajas propiedades mecánicas, lo cual puede ser debido a la forma cúbica de los cristales de $\mathrm{NaCl}$ utilizados. A fin de aminorar dicho efecto, en otros casos se han utilizado, en su lugar, partículas esféricas de carbamida o urea $\left(\left(\mathrm{NH}_{2}\right)_{2} \mathrm{CO}\right)$, la cual es removida previamente a la

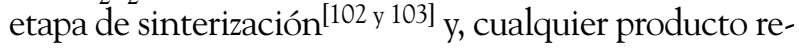
manente puede ser removido durante dicha etapa,

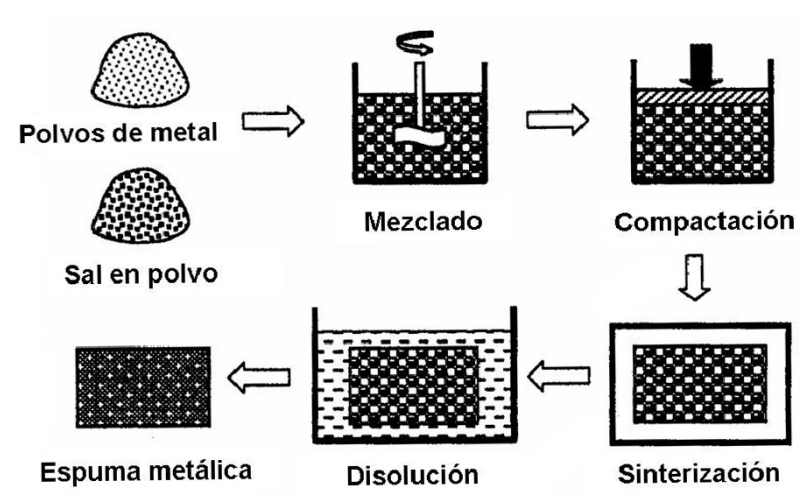

Figura 3. Sinterización de polvos metálicos y rellenos removibles ${ }^{[98]}$.

Figure 3. Metallic powder and space holders sintering ${ }^{[98]}$. 
evitando así la formación de productos frágiles ${ }^{[106]}$. Este novedoso método ha sido trabajado bastante por Zhao et al., quienes obtuvieron esponjas con porosidades entre 50 y $80 \%$ y determinaron que, dependiendo de la distribución de las partículas esféricas de carbamida, se podía controlar, más fácilmente, la morfología de los poros en la esponja ${ }^{[102]}$. Por otro lado, Bram et al. describen como exitoso el uso de carbamida para la fabricación de esponjas de titanio, acero inoxidable 316L y superaleaciones base níquel ${ }^{[104}$ y 105]. En lo que se refiere a la fabricación de esponjas de titanio, Laptev et al. han utilizado polvos de carbamida mezclados con bicarbonato de amonio $\left(\left(\mathrm{NH}_{4}\right) \mathrm{HCO}_{3}\right)$ comprimidos ${ }^{[106]}$. Así mismo, para la obtención de acero inoxidable 316L mediante esta técnica, se conoce el trabajo realizado por Bakan, utilizando partículas de carbamida aglomeradas con una emulsión de polimetilmetacrilato (PMMA), donde la remoción de carbamida con agua permite obtener muestras con una porosidad de un $70 \%$ vol. ${ }^{[107]}$ y otro estudio realizado por Nishiyabu et al. , quienes emplearon partículas esféricas de polimetilmetacrilato aglomeradas con cera parafínica y poliacetal[ ${ }^{[109]}$. Por su parte, Zhao et al. han utilizado una variante de éste método denominado LCS (Lost Carbonate Sintering) para fabricar esponjas de cobre con $\mathrm{K}_{2} \mathrm{CO}_{3}$, obteniéndose porosidades entre 50 y $85 \%{ }^{[109]}$. Por otro lado, el Fraunhofer IFAM ha fabricado esponjas de titanio mediante el uso de gránulos de polímero como space holders, utilizando en este caso la remoción química a temperaturas de alrededor de $130{ }^{\circ} \mathrm{C}{ }^{[110]}$. A continuación se señalan las ventajas y desventajas de este proceso.

\subsubsection{Ventajas}

- Tamaño y distribución de poros controlado por selección de los granos de la preforma removible (powder space holder).

- Flexibilidad en las aleaciones metálicas a usar.

- Obtención de componentes en su forma final, que no sean demasiado complicados.

- Permite la fabricación de esponjas de alta calidad y propiedades reproducibles.

- Proceso con buena relación costo-beneficio.

- Obtención de altas porosidades.

- Fácil remoción y casi total del bicarbonato de amonio y la carbamida por procesos térmicos.

— Posibilidad de disolución de la carbamida en agua.

\subsubsection{Desventajas}

- Durante la remoción del bicarbonato de amonio y la carbamida se liberan gases ambientalmente dañinos.
- Largo tiempo de disolución del molde.

- Esponjas obtenidas pueden tener bajas propiedades mecánicas.

- Posibilidad de corrosión en el metal matriz de la esponja por sal remanente.

- A altas temperaturas de sinterización, se incrementa la tendencia a la evaporación de elementos de aleación y la fusión parcial.

- Dificultad para obtener una morfología homogénea de poros.

- El óxido presente en el Al no permite una mayor unión de partículas durante la sinterización.

- Proceso relativamente costoso.

\subsection{Sinterización de polvos o fibras metálicas}

La empresa GKN Sinter Metals Filter GMBH produce componentes empleando este sistema. Es un proceso basado en la pulvimetalurgia, desde la obtención de los polvos, la mezcla, compactación y sinterización de los mismos. Además de polvos, también es posible el uso de fibras resistentes a la corrosión y a la temperatura, elaboradas en aceros inoxidables, aleaciones de titanio y aleaciones base níquel ${ }^{[111]}$. Contrariamente a los procesos anteriores, existen dificultades para la obtención de esponjas de aleaciones de aluminio, debido a que la capa de óxido superficial que cubre los polvos de este metal no permite una sinterización adecuada ${ }^{[112]}$. En la figura 4 se puede ver el proceso, de forma esquemática. Se puede añadir rellenos que son eliminados en la etapa de sinterización, alcanzando porcentajes de porosidad entre 40 y $85 \%$ ) [113], con densidad relativa en un promedio entre 0,6 y 0,8 y que puede ser más baja para tamaños de poro de 0,5 a 200 $\mu{ }^{[111]}$. Una variante del proceso de sinterización de polvos está concebida dentro del método de presión en caliente por corriente de pulsos, el cual hace uso del sistema de sinterización denominado spark plasma sintering (SPS) [114 y 115] o pulsed electric current sintering (PECS) ${ }^{[116]}$. Este último método permite la sinterización efectiva de materiales con porosidad gradual, compuestos intermetálicos y materiales nanocristalinos. Algunas ventajas y desventajas del proceso de sinterización de partículas o fibras metálicas se relacionan a continuación.

\subsubsection{Ventajas}

- Posibilidad de utilizar fibras metálicas para el aumento de la porosidad

- Sinterización de partículas a baja temperatura mediante pulsos de corriente eléctrica. 


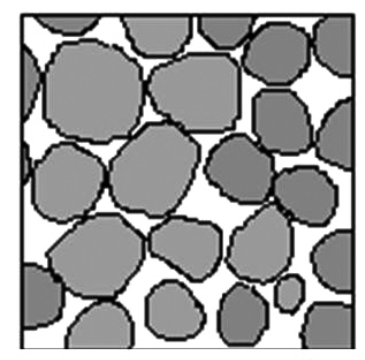

Polvos o fibras metálicas

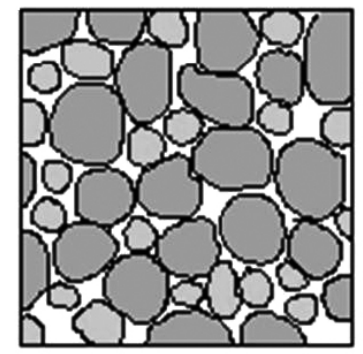

Polvos o fibras metálicas con adición de rellenos que descomponen a alta temperatura

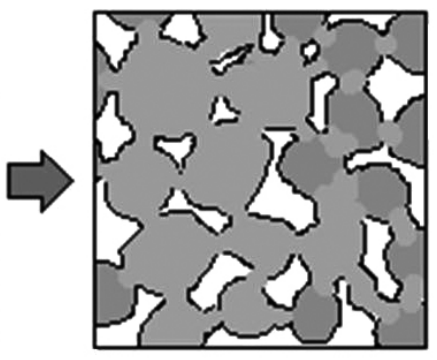

Sinterización

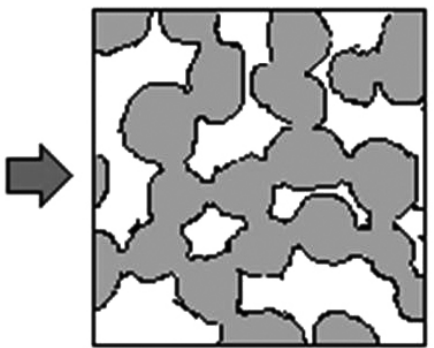

Esponja resultante
Figura 4. Sinterización de polvos o fibras metálicas.

Figure 4. Metallic fibers or powder sintering.

- Posibilidad de obtener gradientes de porosidad en un mismo material.

\subsubsection{Desventajas}

- Requiere alta pureza de los polvos utilizados.

- Problemas para procesar esponjas de aluminio.

- Porosidades relativamente bajas.

\subsection{Métodos para la fabricación de espumas a base de esferas huecas metálicas}

Es posible obtener estructuras altamente porosas utilizando esferas huecas individuales, que pueden ser unidas sólo por efectos difusivos o, en algunos casos, son sometidas a procesos de unión, simplemente, llenando los intersticios con polvos metálicos o suspensiones compuestas por metal y aglomerantes que, posteriormente, son sinterizados. También, es posible hacerlo infiltrando con metal líquido los espacios dejados entre las esferas. Una de las ventajas de las estructuras de esferas huecas es que la distribución del tamaño de poros no es aleatoria, por lo que las propiedades mecánicas y físicas de dichas estructuras pueden ser, por tanto, más predecibles que las de espumas "reales" con un tamaño de poro aleatorio. Por otra parte, puede ser utilizado un amplio intervalo de materiales disponibles en polvos metálicos para el proceso: superaleaciones base níquel, cobre, titanio, acero e intermetálicos. Las estructuras a base de esferas huecas encuentran utilidad en aplicaciones de alta temperatura $^{[111}$ y 112], en la industria automotriz y en aplicaciones de absorción de sonido ${ }^{[117]}$. Sin embargo, aún no está claro si el alto costo de cualquiera de los procesos para la obtención de espumas a base de esferas huecas se compensa lo suficiente con las mejores propiedades en relación con las de otras espumas estocásticas $^{[118]}$. A continuación se hará una breve reseña de algunos de los procesos empleados para la elaboración de espumas a base de esferas huecas, donde los dos primeros procesos se denominan métodos para la elaboración de esferas sin núcleo y los tres procesos posteriores se denominan procesos con núcleo.

\subsubsection{Atomización por gas}

Proceso que genera pequeñas cantidades (típicamente entre 1-5\%) de partículas huecas debido al atrapamiento de gas en los ligamentos líquidos. Mediante métodos de separación apropiados, estas partículas, pueden separarse y consolidarse para producir estructuras de esferas huecas con diámetros entre 500-1.000 $\mu \mathrm{m}$ y con espesor de pared entre 100 y $300 \mu \mathrm{m}$. Por este método se han fabricado con éxito estructuras de $\mathrm{Ti}-6 \mathrm{Al}-4 \mathrm{~V}$ y superaleciones base níquel ${ }^{[111]}$.

\subsubsection{Barbotina atomizada coaxialmente}

El sistema desarrollado por el Instituto Tecnológico de Georgia consiste en crear burbujas mediante un sistema de boquilla coaxial, en la que un gas pasa a través del orificio interno y realiza el soplado de una solución o barbotina compuesta por polvos metálicos, cerámicos o hidruros, un aglomerante y dispersantes, que sale por la parte externa de la boquilla ${ }^{[111}$ y 119], como se describe en la figura 5. Una vez formadas las burbujas, la fase líquida es eliminada por evaporación propiciando la coalescencia de las partículas sólidas, dando a lugar a esferas de cierta consistencia. Posteriormente, las esferas son tratadas térmicamente para eliminar la fase líquida restante y otros componentes. Una producción típica de esferas puede estar entre 3.000 y 15.000 esferas $/ \mathrm{min}$, las cuales poseen diámetros entre 1 y $6 \mathrm{~mm}$ y espesores entre 40 y 200 
PROCESOS DE FABRICACIÓN DE METALES CELULARES. PARTE II: VÍA SÓLIDA, DEPOSICIÓN DE METALES, OTROS PROCESOS MANUFACTURING PROCESSES OF CELLULAR METALS. PART II. SOLID ROUTE, METALS DEPOSITION, OTHER PROCESSES

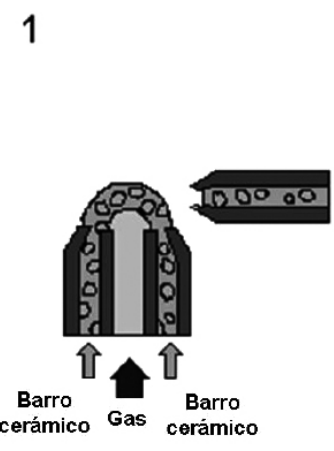

4

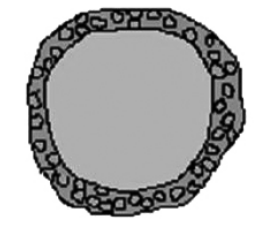

Mediante calentamiento se elimina el aglomerante y las particulas sólidas se unen
2

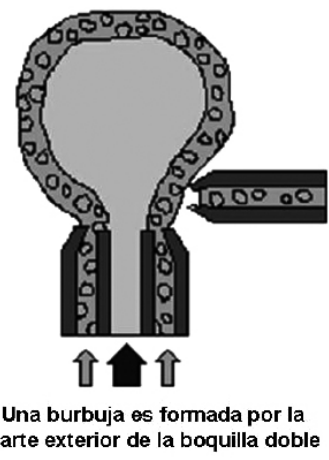

5

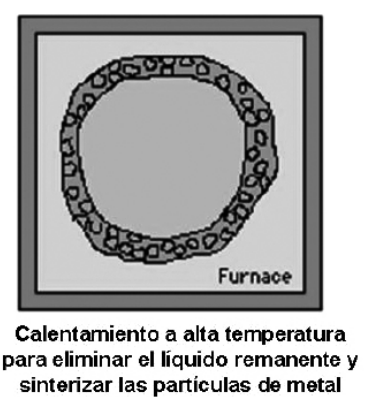

3

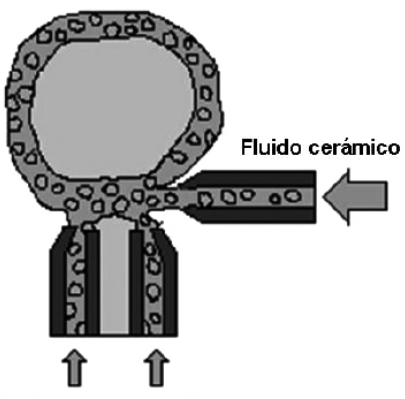

Mediante una boquilla transversal adiciona el fluido que cierra la burbuja

6

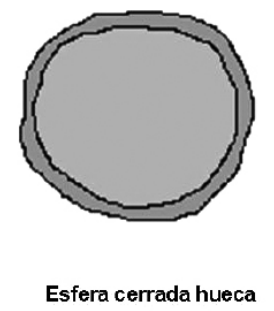

Figura 5. Fabricación de esferas huecas por inyección de gas y barro cerámico.

\section{Figure 5. Hollow spheres manufacturing by gas and ceramic slurry injection.}

$\mu \mathrm{m}{ }^{[120]}$. Además, mediante el empleo de mezclas de $\mathrm{Fe}_{2} \mathrm{O}_{3} / \mathrm{Cr}_{2} \mathrm{O}_{3}$ en la barbotina, se pueden crear esferas de acero inoxidable mediante la reducción de las mismas en atmósfera de hidrógeno/argón a $1.400^{\circ} \mathrm{C}[121]$.

\subsubsection{Esferas de poliestireno recubiertas por suspensión de polvos metálicos}

En el sistema patentado por Jaeckel en 1987, y desarrollado por el IFAM en Dresden, se inicia fluidizando las esferas en un flujo de aire mientras se recubren simultáneamente por polvos metálicos en suspensión, luego, se procede al secado del recubrimiento y, posteriormente, al tratamiento térmico para la eliminación del núcleo de poliestireno expandido ${ }^{[119}$ y 122]. El proceso de sinterización depende del tipo de polvos metá-

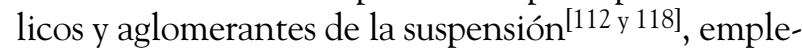
ándose, por ejemplo, temperaturas de sinterización de alrededor de $1.250^{\circ} \mathrm{C}$ y $1.120^{\circ} \mathrm{C}$ en atmósfera reductora para la obtención de esferas huecas de acero inoxidable $316 \mathrm{~L}$ y de hierro, respectivamente ${ }^{[117]}$. Aunque es posible producir esferas de diferentes materiales, el acero inoxidable 316 ha sido uno de los materiales más trabajados por el IFAM, obteniendo esferas con diámetros entre 0,5-10 $\mathrm{mm}$ y espesores de pared entre 10 y $1.000 \mu \mathrm{m}^{[111]}$. El titanio, también ha sido utilizado para la fabricación de esferas huecas, tal como lo escribe Dunand en su revisión de procesos ${ }^{[123]}$. En la figura 6 se esquematiza el proceso descrito y en la figura 7 muestra una variante de este proceso, donde las esferas de poliestireno se recubren mediante inmersión directa en la suspensión.

\subsubsection{Esferas huecas fabricadas por sinterización}

En este caso, se sumergen partículas esféricas de hierro en una solución de $\mathrm{CuSO}_{4}$, lo que da origen a una reacción de electroless por desplazamiento mediante la cual se deposita cobre puro sobre las partículas y, simultáneamente, el núcleo de hierro está siendo disuelto, dando lugar a esferas huecas de cobre con diámetros promedio de 500-750 $\mu \mathrm{m}$. Las partículas de cobre son tratadas térmicamente para mejorar su resistencia. Este tipo de partículas son aglomeradas en moldes cerámicos, mediante sinterización para formar estructuras que exhiben, en general, buena unión entre partículas con densidades relativas cercanas al $20 \%{ }^{[111]}$.

\subsubsection{Esferas de poliestireno expandido recubiertas galvánicamente}

Para este proceso, las esferas de poliestireno expandido son recubiertas galvánicamente mediante un 


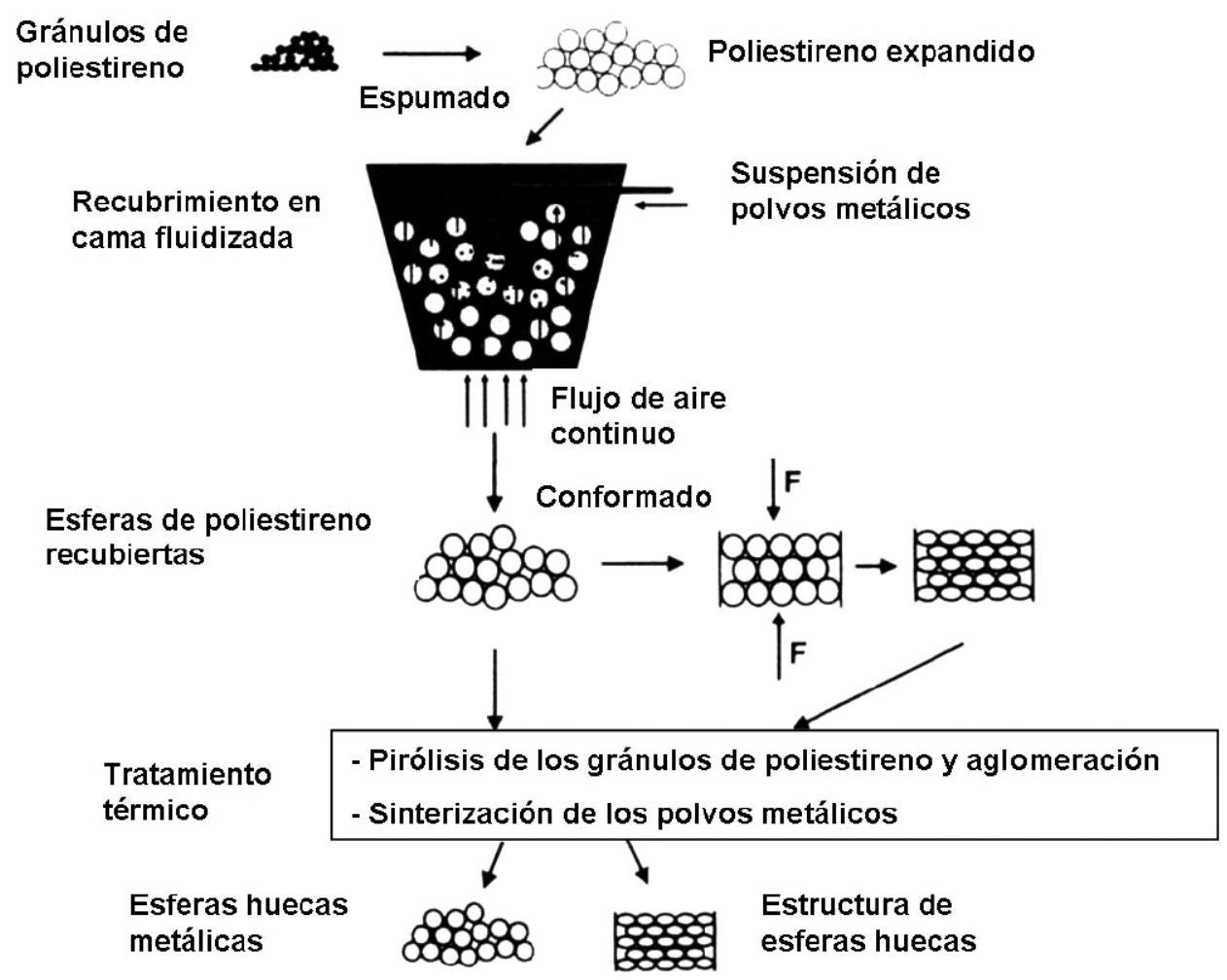

Figura 6. Fabricación de esferas huecas metálicas mediante recubrimiento en lecho fluidizado[117].

Figure 6. Metal hollow spheres manufacturing by fluidized bed covering[117].

1

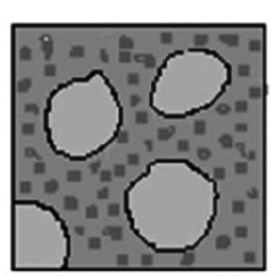

Inmersión de esferas de poliestireno en el barro cerámico

\section{3}

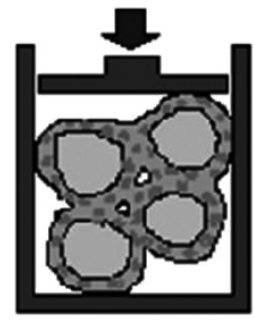

Las esferas son comprimidas mecánicamente
2

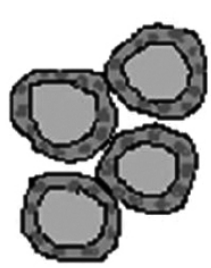

Tratamiento térmico conduce a la remoción de la humedad del barro

4 cerámico

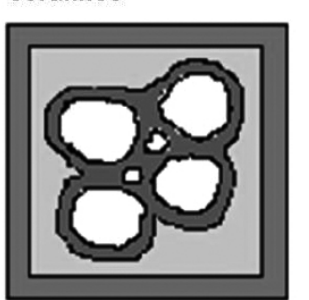

El compacto es quemado para remover el aglomerante y el poliestireno; y para unir las esferas.

Figura 7. Fabricación de esferas huecas metálicas mediante recubrimiento por inmersión.

Figure 7. Metal hollow spheres manufacturing by immersion covering.

proceso electroquímico, actuando como un núcleo que será removido térmicamente. De esta forma, es posible obtener esferas con excelente uniformidad en el espesor de las paredes, las cuales pueden llegar a ser de tan solo unas micras y con diámetros que oscilan entre los 2 y $10 \mathrm{~mm}$. El proceso está limitado a metales útiles para la deposición galvánica (p.ej., cobre y níquel). Para la elaboración de estructuras metálicas, las esferas obtenidas pueden ser unidas mediante procesos de difusión o soldadura blanda ${ }^{[111]}$.

\subsection{Proceso con núcleo de baja densidad (LDC process)}

Denominado proceso con núcleo de baja densidad, de su sigla en inglés Low Density Core-LDC process $^{[111]}$. Esta técnica comienza con la compactación de los polvos para la formación de un material precursor, proceso durante el cual se atrapa el gas en el material para luego ser expandido por calentamiento ${ }^{[124]}$. El componente obtenido posee una porosidad sin conexión entre 20 y 40 \% y, según Elzey y Wadley, no es posible obtener una porosidad mayor al $50 \%$ [125]. Para la fabricación de espumas de titanio ${ }^{[126-129]}$, el polvo del metal es colocado dentro de un recipiente 
PROCESOS DE FABRICACIÓN DE METALES CELULARES. PARTE II: VÍA SÓLIDA, DEPOSICIÓN DE METALES, OTROS PROCESOS MANUFACTURING PROCESSES OF CELLULAR METALS. PART II. SOLID ROUTE, METALS DEPOSITION, OTHER PROCESSES

que contiene gas argón a una presión alrededor de 5 atm, el cual genera la compresión isostática en caliente, requerida para lograr la densificación de la estructura. Posteriormente, el material es convertido en una espuma mediante un tratamiento térmico que provoca la expansión lenta de los poros. En el material obtenido no existe conexión en la porosidad, los diámetros de poro oscilan entre 10 y $100 \mu \mathrm{m}$ y la densidad relativa puede estar entre 0,65-0,5. Este método ha sido aplicado, especialmente, para la fabricación de estructuras porosas de titanio para las aeroestructuras de la Boeing (EE. UU.) ${ }^{[130]}$. No obstante, también pueden ser fabricadas estructuras en aleaciones de cobre y aleaciones base níquel. En la figura 8, Banhart muestra un esquema del proceso planteado por Martín, R.L. y Lederich, R.J.

\subsection{Proceso de laminación acumula- tiva (ARB process)}

Kitazono et al. desarrollaron un novedoso proceso denominado Acumulative Roll-Bonding (ARB) para la obtención de espumas metálicas con porosidades entre $40 \%$ y $60 \%$. En dicho proceso, el material de inicio consiste en polvos del agente espumante $\left(\mathrm{TiH}_{2}\right)$ que se dispone entre láminas de aluminio (p.ej., A4045, A1050); este conjunto es laminado en varios ciclos, como se describe en la figura $9 \mathrm{y}$, finalmente, la preforma obtenida es espumada a alta temperatura ${ }^{[131}$ y 132$]$.

\subsection{Espumado de barbotinas (Sinteri- zación - Reacción)}

Por medio de este proceso, denominado también Slip Reaction Foam Sintering (SRFS) ${ }^{[133]}$, las esponjas me-

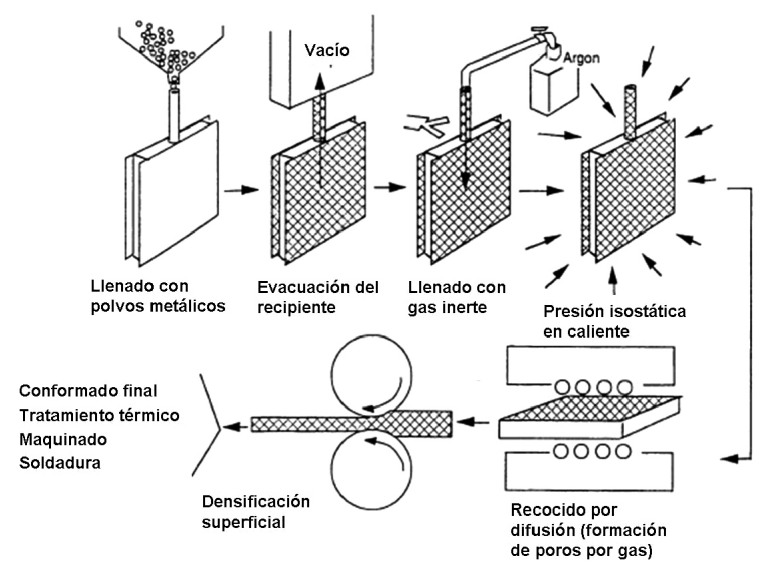

Figura 8. Proceso LDC (Boeing) [112].

Figure 8. LDC Process (Boeing) [112].

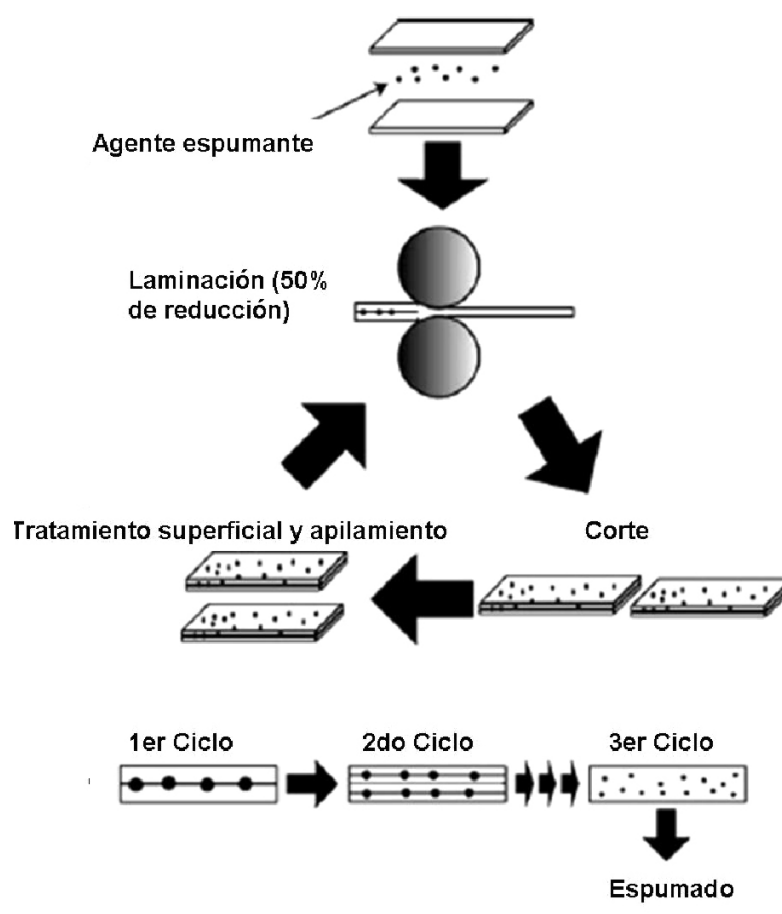

Figura 9. Proceso $A R B^{[131]}$.

Figure 9. ARB Process ${ }^{[131]}$.

tálicas se producen mediante la preparación de una barbotina compuesta por pasta de polvos metálicos, agentes de espumado y algunos aditivos reactivos y dispersantes. La barbotina es mezclada y vaciada dentro de un molde, y después es secada a elevadas temperaturas. Bajo la influencia de los aditivos y del agente de espumado, la pasta se torna viscosa y comienza a expandirse mientras el gas comienza a evolucionar. Finalmente, la pasta es secada y sinterizada para producir una esponja de metal con una resistencia considerable ${ }^{[24]}$. Algunas esponjas han sido producidas a partir de polvos de aluminio, usando ácido fosfóri$\mathrm{co}^{[133]} \mathrm{u}$ ortofosfórico con hidróxido de aluminio o ácido hidroclórico como agente espumante. En el caso de las esponjas de acero inoxidable o base níquel se utilizan polvos metálicos puros altamente reactivos, tales como carbonilo de hierro o carbonilo de níquel ${ }^{[134]}$. Mitsubishi obtiene por esta vía esponjas de acero inoxidable, cobre y níquel con porosidades entre el 70 y $97 \%$ [135]. Sin embargo, cabe observar que no se considera un método atractivo para la fabricación de esponjas, debido a la baja resistencia que presenta la estructura metálica resultante [111 y 112].

Una variable del proceso la constituye la composición de la barbotina a base de polvos metálicos y aglomerante, mezclados con cera fundida y agente dispersante, obteniéndose una emulsión que será solidificada (tornándose como un gel); luego, la cera 
es removida y el polvo metálico es sinterizado para obtener la espuma metálica, como se muestra en la figura $10^{[136]}$. Existe una forma alternativa para el uso de ésta técnica, que consiste en sumergir una espuma polimérica de poros abiertos en una pasta que contiene una mezcla de polvos de plata y óxidos de plata; después, el polímero es retirado térmicamente de la espuma cubierta y las partículas de polvo metálico comienzan a sinterizar formando una estructura metálica celular rígida ${ }^{[12]}$.

\subsection{Espumado por reacción de combustión}

El proceso de espumado por reacción de combustión consiste, esencialmente, en realizar una mezcla de polvos metálicos compactados que se calientan para dar inicio a la reacción entre los polvos elementales y, así, dar lugar a la formación de materiales intermetálicos, como se muestra en la figura 11. El proceso de síntesis por combustión se caracteriza por ser un método sencillo y con un tiempo corto de procesamiento, útil para la obtención de materiales porosos de compuestos intermetálicos tales como el TiNiSMA (aleación con memoria de forma) ${ }^{[137]}, \mathrm{Ni}-\mathrm{Al}$, $\mathrm{Ni}_{3} \mathrm{Al}_{\text {y }} \mathrm{NiAl}_{3}{ }^{[94}$ y 138]. Para el procesamiento de los

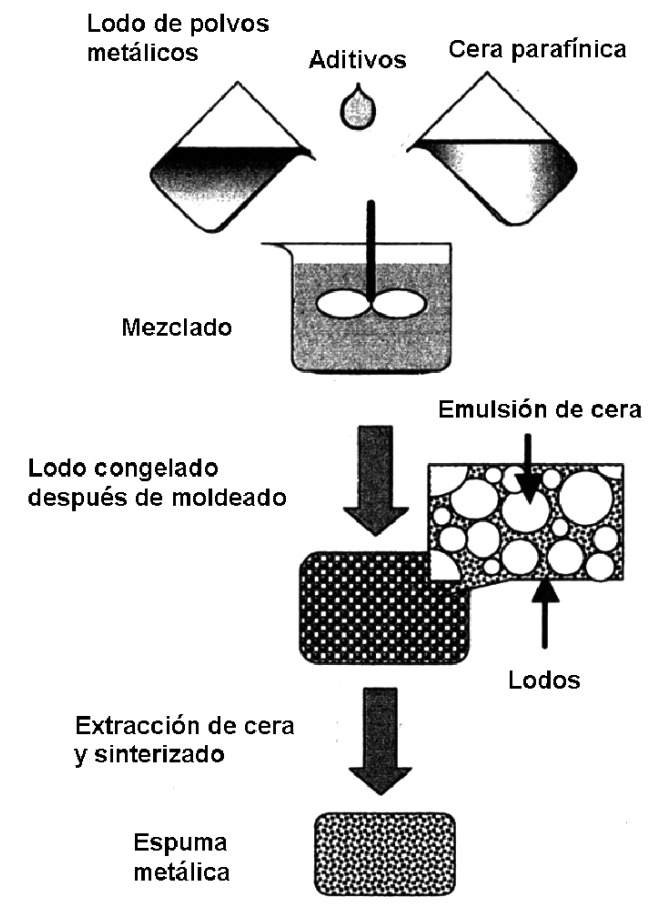

Figura 10. Espumado de lodos ${ }^{[136]}$.

Figure 10. Slurries foaming ${ }^{[136]}$.

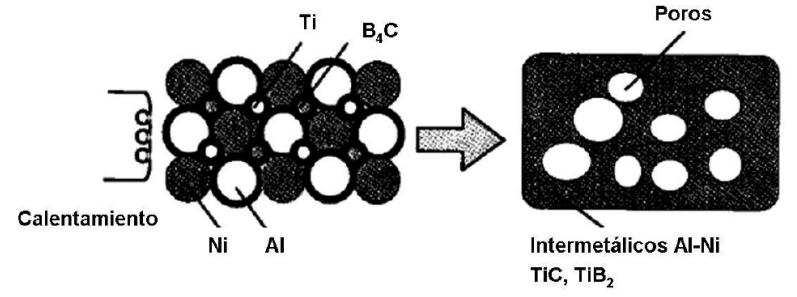

Figura 11. Espumado por reacción de combustión ${ }^{[138]}$.

Figure 11. Combustion reaction foaming[138].

tres últimos, es común el uso de $\mathrm{B}_{4} \mathrm{C}$ como agente catalizador de la reacción y el $\mathrm{TiC}$ como agente absorbente de calor, los cuales se mezclan con los polvos elementales de aluminio y de níquel que serán sometidos a reacción. La porosidad de la espuma se incrementa efectivamente por efecto del $\mathrm{B}_{4} \mathrm{C}$, a un $80 \%$ y el $\mathrm{TiC}$, por el contrario, causa una disminución de la misma. Existen varios aspectos a tener en cuenta para el procesamiento de esponjas de $\mathrm{Ni}_{3} \mathrm{Al}$ :

- el uso de $\mathrm{B}_{4} \mathrm{C}$ aumenta la temperatura de combustión promoviendo la generación de gas,

- la formación de partículas de $\mathrm{NiAl}_{3}$, que incrementan la viscosidad a alta temperatura y

- el uso de polvos de níquel promueve la reacción por combustión ${ }^{[139]}$. Sin embargo, cuando se utiliza agente espumante, los poros tienden a ser de mayor tamaño y con morfología irregular ${ }^{[140]}$.

\subsection{Espumado de metales mediante uso de espumas cerámicas precursoras (CFP)}

La producción de metales celulares mediante el uso de precursores a base de espumas cerámicas (Ceramic Foam Precursors, CFP) es un proceso que ha sido utilizado exitosamente por Verdooren et al. ${ }^{[141}$ y 142] $\mathrm{pa}-$ ra la obtención de espumas de metales férreos, evadiendo las dificultades que conlleva su elaboración por otros métodos. En este método, la espuma de hierro es producida mediante el espumado de una mezcla de óxidos de hierro $\left(\mathrm{Fe}_{2} \mathrm{O}_{3}\right)$ con otros aditivos, para pasar luego a una etapa de reducción completa del precursor cerámico a una temperatura alrededor de los $1.240{ }^{\circ} \mathrm{C}$ en una mezcla de $4 \% \mathrm{H}_{2}$-Argón. Este método permite obtener espumas metálicas de poros cerrados con densidad relativa de alrededor de 0,23 y tamaños de poros entre 0,5-2 $\mathrm{mm}$. El espumado se realiza a temperatura ambiente, las materias 
PROCESOS DE FABRICACIÓN DE METALES CELULARES. PARTE II: VÍA SÓLIDA, DEPOSICIÓN DE METALES, OTROS PROCESOS MANUFACTURING PROCESSES OF CELLULAR METALS. PART II. SOLID ROUTE, METALS DEPOSITION, OTHER PROCESSES

primas son relativamente de bajo costo y los equipos requeridos son de tipo estándar y de amplio uso a nivel industrial, por lo que el proceso permite ser implementado a gran escala y para la fabricación de formas complejas. El proceso es adaptable a un amplio intervalo de composiciones de aceros y de metales de alto punto de fusión que por otros métodos representarían un alto costo económico ${ }^{[143]}$.

\section{TÉCNICAS DE DEPOSICIÓN}

\subsection{Electrodeposición}

Es el proceso empleado por Sumitomo Electric Industries (Celmet), Japón, Sorapec (Metapore) y Recemat ${ }^{[144]}$. El proceso consiste en hacer conductora una esponja de polímero, comúnmente poliuretano, por inmersión en un baño coloidal de grafito o negro de carbono o por vaporización de una fina capa de metal, para ser recubierta, después, por un metal mediante electrodeposición o deposición galvánica. Posteriormente, se somete a un proceso de sinterización y eliminación del modelo (poliuretano) por tratamiento térmico o químico. Es posible obtener componentes de poro abierto, de aproximadamente $95 \%$ de porosidad, con tamaño de celda entre 400 y $5.000 \mu \mathrm{m}$ y con densidades en un intervalo de $0,2-0,5 \mathrm{~g} / \mathrm{cm}^{3}[1$ y 112$]$. En la figura 12 se puede ver el procedimiento para obtener una esponja por electrodeposición. Los metales para éste proceso están limitados al níquel, aleaciones $\mathrm{Ni}$ - $\mathrm{Cr}, \mathrm{Cu}, \mathrm{Ag}$, y $\mathrm{Sn}{ }^{[111]}$. Algunas ventajas y desventajas se relacionan a continuación.

\subsubsection{Ventajas}

— Es posible reutilizar los electrolitos y los ánodos consumibles.

- Permite producción de placas grandes de composición específica.

- Obtención de estructuras con un buen nivel de homogeneidad en la porosidad.

\subsubsection{Desventajas}

- Posibilidad de discontinuidades en el espesor de las capas depositadas en la preforma.

- Proceso costoso y requiere mayor control.

- Sólo pocos metales pueden ser depositados superficialmente por medios electrolíticos.

\subsection{Deposición en fase gaseosa}

El proceso de deposición en fase gaseosa es similar al descrito para la electrodeposición en cuanto al uso de una esponja polimérica que es eliminada por quemado luego de ser recubierta por deposición de

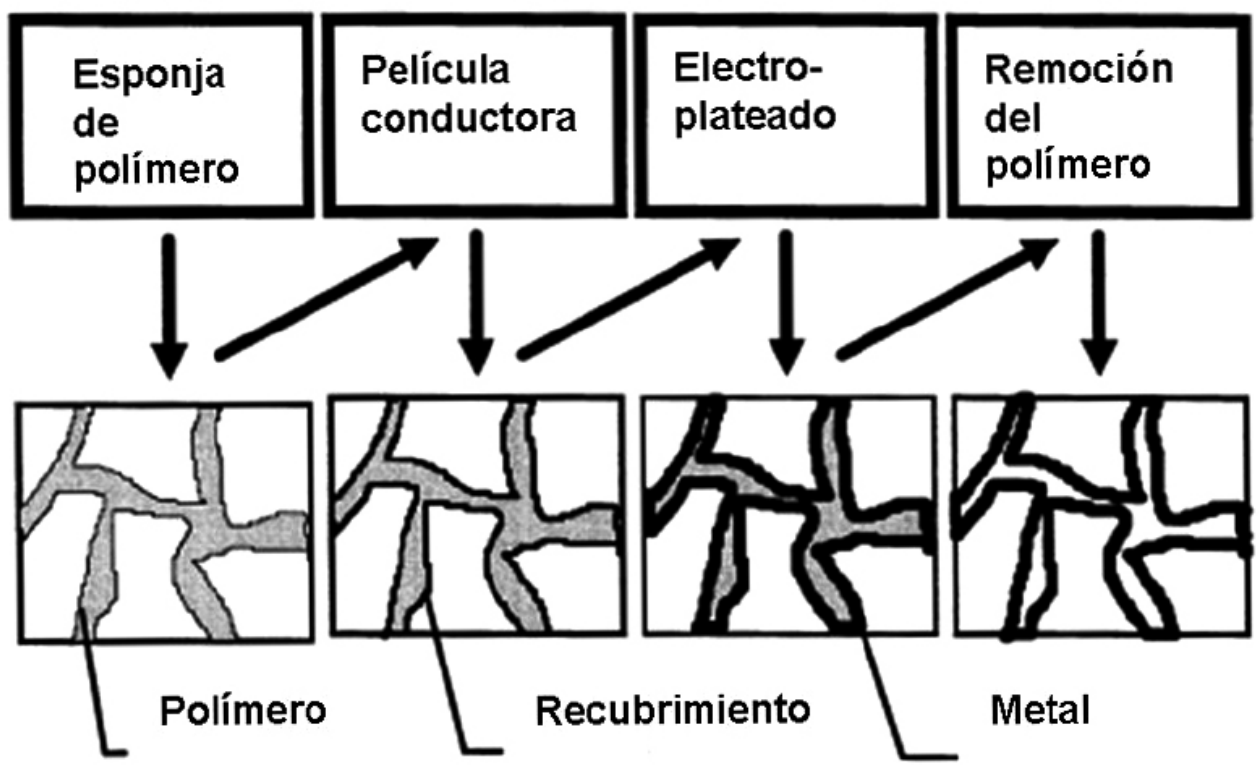

Figura 12. Producción de esponjas metálicas por electrodeposición ${ }^{[112]}$.

Figure 12. Metallic sponges production by electrodeposition ${ }^{[112]}$. 
metales gaseosos o compuestos metálicos gaseo$\operatorname{sos}^{[112]}$. Algunas de las características de este tipo de esponjas son: sus valores de densidad, desde 0,2$2,6 \mathrm{~g} / \mathrm{cm}^{3}$, la pureza del material que llega a $\sim 99,97$ $\%$ y la porosidad que está en un intervalo del 70 . 98 \% vol. Para la fabricación por CVD (Deposición Química en fase Vapor) se utiliza como precursor carbonilo de níquel, que se descompone a $120^{\circ} \mathrm{C}$, depositándose el níquel sobre un sustrato de poliuretano que luego será retirado mediante calentamiento y, finalmente, se sinteriza el níquel depositado para incrementar su ductilidad. En este proceso, la densidad relativa está determinada por el espesor del metal depositado en el polímero y los tamaños de celda oscilan entre los 450-3.200 $\mu \mathrm{m}$ [145]. Inco Ltd., en Canadá, utiliza este método para la producción de esponjas de níquel comercializadas con el nombre de Incofoam, cuya aplicación establecida es como material para electrodos de batería $^{[146]}$. Existe la posibilidad de depositar aleaciones de otros metales diferentes al níquel, tales como superaleaciones del tipo $\mathrm{Ni}-\mathrm{Al}$ y $\mathrm{Ni}-\mathrm{Cr}-\mathrm{Al}{ }^{[147]}$ y es posible la deposición de intermetálicos como $\mathrm{Ni}_{3} \mathrm{Al} / \mathrm{NiAl}$ por difusión reactiva de aluminio. En la figura 13 se describe el proceso de deposición en fase gaseosa. Más adelante se indican algunas de las ventajas y desventajas del proceso descrito.

\subsubsection{Ventajas}

- Alta pureza de los metales depositados

- Obtención de espumas de muy baja densidad

\subsubsection{Desventajas}

- Proceso costoso

- Mayor control de parámetros de proceso

\section{CONCLUSIONES}

En la actualidad, existen diferentes métodos para la fabricación por vía sólida, líquida o gaseosa de espumas y de esponjas metálicas. Aunque es posible obtener un material celular casi en cualquier metal puro o aleación, el aluminio es el metal de mayor desarrollo tanto a nivel académico-científico como industrial, debido a que sus características particulares lo hace adecuado para muchas de las aplicaciones propuestas y; en muchos casos, minimiza algunas de las dificultades que pueden presentar otros metales como las aleaciones de hierro (punto de fusión), el magnesio (reactividad), entre otros. Por otra parte, es

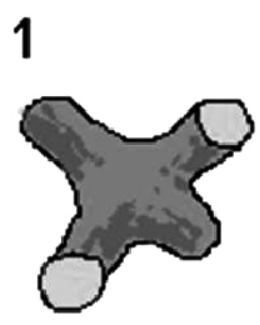

Polímero con celdas abiertas (Se muestra un nodo sencillo)

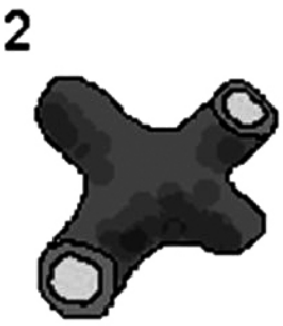

Recubrimiento con negro de carbono

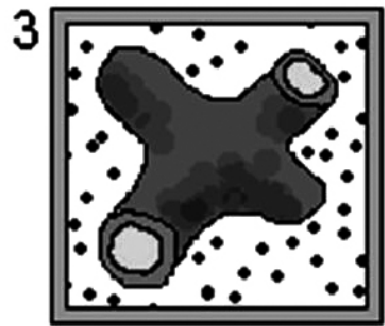

Atomización con gas de Carbonilo de Níquel

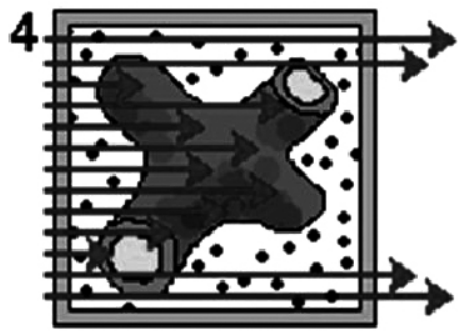

Calentamiento por radiación infrarroja
5

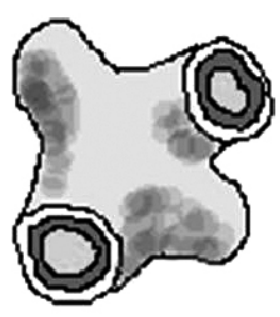

Esponja de polímero recubierta con una capa de Níquel
6

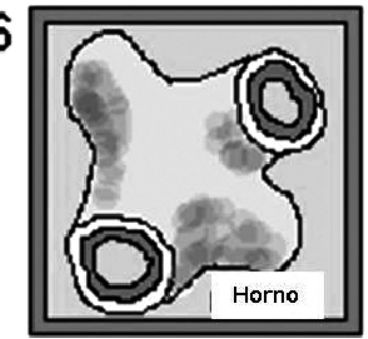

Sinterización de la esponja y remoción del polímero a alta temperatura

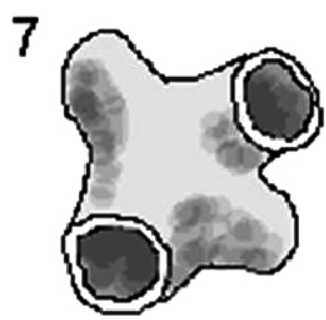

Esponja metálica de poros abiertos con paredes de celda huecas

Figura 13. Proceso de deposición de fase gaseosa.

Figure 13. Gas deposition process. 
posible observar que la mayoría de los procesos requieren de equipos e insumos costosos, que además de encarecer el valor del material celular obtenido dificulta en algún grado su implementación a nivel industrial.

De las vías de fabricación revisadas para la obtención de esponjas y espumas metálicas, se destaca el nivel de evolución alcanzado por la vía pulvimetalúrgica para la fabricación de espumas, evidenciándose esto en la gran cantidad estudios que se encuentran en la literatura acerca de las fenomenologías asociadas a su producción y con respecto a la caracterización de sus propiedades y en los desarrollos que de manera satisfactoria vienen siendo empleados en aplicaciones tecnológicas de sectores como el automotriz y el aeronáutico. Así mismo, el proceso llevado a cabo mediante el uso de preformas de poliuretano sobresale entre los métodos para la obtención de esponjas metálicas, tanto por vía líquida como por deposición metálica.

A pesar del notable desarrollo que ha alcanzado el procesado de materiales celulares, en la última déca$\mathrm{da}$, siguen existiendo varias preguntas en cuanto a su viabilidad económica e industrialización, lo que conlleva la necesidad de continuar investigando en el planteamiento de nuevos procesos y en la optimización de las vías existentes, de las cuales algunos procesos por fundición y otros por pulvimetalurgia presentan el mayor potencial para la producción en masa a costos razonables.

\section{REFERENCIAS}

[1] J. Banhart y J. Baumeister, Proc. Porous and cellular materials for structural applications, Vol. 521, D.S. Schwartz, D.S. Shih, A.G. Evans and H.N.G. Wadley (Eds.), MRS, Warrendale, Pennsylvania, USA, 1998, pp. 121-132.

[2] A. G. Evans, J. W. Hutchinson y M. F. Ashby, Curr. Opin.Solid St.Mater. Sci. 3 (1998) 288303.

[3] K. Stöbener; J. Baumeister; D. Lehmhus; H. Stanzick y V. Zöllmer. Proc. Int. Conf. Advanced Metallic Materials, Smolenice, Eslovaquia, 2003, pp. 281-286.

[4] J. Banhart, J. Baumeister y M. Weber, Metal Powder Report (1997) 38-41.

[5] G. Rausch, J. Baumeister, D. Lehmhus, H. Stanzick, K. Stöbener, M. Wichman, N. Zimmer y V. Zöllmer, Fraunhofer Institute for Manufacturing and Advance Materials. $<$ http://www.ifam.fhg.de/2801/leichtbauwerkstoffe/metallschaeume/design/design_guidelines_english_V14.pdf $>$ (9 enero 2007)
[6] R. Surace, L.A.C. De Filippis, A.D. Ludovico y G. Boghetich, Proc. 5th International DAAAM Baltic Conference, Tallinn, Estonia, 2006, pp. 301-306.

[7] K. Stöbener, J. Baumeister, G. Rausch y M. Busse, Metal-Powder.Net, MPR (2005) 13-16.

[8] P. Schäffler, G. Hanko, H. Mitterer y P. Zach, Proc. MetFoam 2007 Porous Metals and Metallic Foams, L.P. Lefebvre, J. Banhart and D.C. Dunand (Eds.), Destech Publications, Inc. Pensylvania, USA, 2008, pp. 7-10.

[9] P. Schäffler y W. Rajner, Proc. Cellmet 2005 Cellular Metals for structural and functional applications. G. Stephani and B. Kieback (Eds.), Fraunhofer IRB Verlag, Dresden, Alemania, 2005. pp. 125-130.

[10] J. Baumeister, Proc. Int. Conf. Metal foams and porous metal structures, J. Banhart, M. F. Ashby, N.A. Fleck (Eds.), MIT Press-Verlag Bremen, Alemania, 1999, pp. 113-118.

[11] R. Braune y A. Otto, Proc. Int. Conf. Metal foams and porous metal structures, J. Banhart, M. F. Ashby, N.A. Fleck (Eds.), MIT Press-Verlag Bremen, Alemania, 1999, pp. 119-124.

[12] V. Gergely, F. Simanãik, T. J. Matthams y T. W. Clyne, Proc. 12th Int. Conf. on Composite Materials, T. Massard \& A. Vautrin (Eds.), Paris, 1999, pp. 1-10.

[13] H. W. Seeliger, Proc. MetFoam 2003 Cellular Metals: Manufacture, properties, applications, J. Banhart, N. A. Fleck, A. Mortensen (Eds.), MIT Press-Verlag, Berlin, Alemania, 2003, pp 5-12.

[14] J. Kováãik, F. Simančík, J. Jerz y P. Tobolka, Proc. Int. Conf. Advanced Metallic Materials, Smolenice, Slovakia, 2003, pp. 154-159.

[15] J. Banhart y F. Baumgärtner, <http://www.hmi. de/people/banhart/B-Conferences/b051_banhart2002.pdf > (9 enero 2007).

[16] S. Vachiants y N. Manukyan, Proc. MetFoam 2003 Cellular Metals: Manufacture, properties, applications, J. Banhart, N. A. Fleck, A. Mortensen (Eds.), MIT Press-Verlag, Berlin, Alemania, 2003, pp. 131-136.

[17] M. Bram, H. P. Buchkremer, A. Laptev y D. Stöver, Proc. 2nd Int. Conf. Cellular metals and metal foaming technology, J. Banhart, M.F. Ashby, N.A. Fleck (Eds.), MIT-Verlag, Bremen, Alemania, 2001, pp. 215-220.

[18] I. Duarte y J. Banhart, Acta Mater. 48 (2000) 2349-2362.

[19] F. Baumgärtner y H. Gers, Proc. Int. Conf. Metal foams and porous metal structures, J. Banhart, M. F. Ashby, N.A. Fleck (Eds.), MIT PressVerlag Bremen, Alemania, 1999, pp. 73-78.

[20] C. Jiménez y M. Malachevsky, Matéria 9 (2004) $80-85$.

[21] C. Kammer, TALAT Lecture 140 (1999) 1-24. 
[22] V. Amigó, D. Busquets y A. Cárcel. Proc. Actas del VI Congreso Nacional de Materiales Compuestos. AEMAC-UPV, Valencia, España, 2005, pp. 247-254.

[23] Ch-J. Yu, H. H. Eifert, J. Banhart y J. Baumeister, Adv. Mater. Process. 11 (1998) 45-47.

[24] J. Banhart. http://www.hmi.de/people/banhart/B-Conferences/b021_banhart1998.pdf. pp. 3-11 (15 enero 2007).

[25] F. Simancík, H.P. Degischer y H. Wörz. Proc. 4th Europ. Conf. Advanced Materials and Processes (Euromat), Vol. IV, Assoc. Italian. de Metall. (Eds.), Venice/Padua, Italy, 1995, pp. 191-196.

[26] J. Banhart y F. Baumgärtner, Handbook of Cellular Materials - Production, Processing, Applications, H.P. Degischer and B. Kriszt (Eds.), Wiley-VCH, Weinheim, Alemania, 2002, pp. 14-21.

[27] Ch-J. Yu, H. H. Eifert, J. Banhart y J. Baumeister, Mater. Res. Innov. 2 (1998) 181-188.

[28] F. Garcia-Moreno, N. Babcsan, J. Banhart, M. Haesche y J. Weise, Proc. Cellular Metals and Polymers, Fürth, Alemania, 2004, R.F. Singer, C. Körner, V. Altstädt, H. Münstedt, Trans. Tech. Publications, Zuerich, Switzerland, 2005, pp. 1-4.

[29] J. Weise, O. Marchetto, M. Haesche, F. GarciaMoreno y J. Banhart, Proc. MetFoam2005 Porous Metals and Metal Foaming Technology, The Japan Institute of Metals (Eds.), Kyoto, Japan, 2005, pp..21-23.

[30] F. Garcia-Moreno, J. Banhart, M. Haesche, K. Vignodhar y J. Weise, Proc. Cellmet 2005 Cellular Metals for structural and functional applications. G. Stephani and B. Kieback (Eds.), Fraunhofer IRB Verlag, Dresden, Alemania, 2005. pp. 244-252.

[31] Y. Chino, H. Nakanishi, M. Kobata, H. Iwasaki y M. Mabuchi, Scripta Mater 47 (2002) 769. 773.

[32] J. Weise, H. Stanzick y J. Banhart, Proc. MetFoam 2003 Cellular Metals: Manufacture, properties, applications, J. Banhart, N. A. Fleck, A. Mortensen (Eds.), MIT Press-Verlag, Berlin, Alemania, 2003, pp. 169-174.

[33] J. Baumeister, J. Weise, A. Jeswein, M. Busse y M. Haesche, Proc. MetFoam 2007 Porous Metals and Metallic Foams, L.P. Lefebvre, J. Banhart and D.C. Dunand (Eds.), Destech Publications, Inc. Pensylvania, USA, 2008, pp. 83-86.

[34] T. Höpler, F. Schörghuber y F. Simančik, Proc. Int. Conf. Metal foams and porous metal structures, J. Banhart, M. F. Ashby, N.A. Fleck (Eds.), MIT Press-Verlag Bremen, Alemania, 1999, pp. 79-82.
[35] F. Simančik y F. Schoerghuber, Proc. Porous and cellular materials for structural applications, D.S. Schwartz, D.S. Shih, A.G. Evans and H.N.G. Wadley (Eds.), MRS Vol. 521, Warrendale, Pennsylvania, USA, 1998, pp. 151 157

[36] N. Babcsán, J. Banhart y D. Leitlmeier, Proc. Intern. Conf. Advanced Metallic Materials, Smolenice, Slovakia, 2003, pp. 5-15.

[37] F. Simančík, N. Mináriková, S. Čulák y J. Kováčik, Proc. Int. Conf. Metal foams and porous metal structures, J. Banhart, M. F. Ashby, N.A. Fleck (Eds.), MIT Press-Verlag Bremen, Alemania, 1999, pp. 105-108.

[38] H-M. Helwig, F. Garcia-Moreno y J. Banhart, Proc. Cellular Metals for structural and functional applications. Cellmet 2005. G. Stephani and B. Kieback (Eds.), Fraunhofer IRB Verlag, Dresden, Alemania, 2005, pp. 253-257.

[39] K. Stöbener, M. Weigend y G. Raush, Proc. MetFoam 2003 Cellular Metals: Manufacture, properties, applications, J. Banhart, N.A. Fleck, A. Mortensen (Eds), MIT Press-Verlag, Berlin, Alemania, 2003, pp. 161-64.

[40] C. J. Yu, H. H. Eifert, M. Knuewer, M. Weber y J. Baumeister, Proc. Porous and cellular materials for structural applications, D.S. Schwartz, D.S. Shih, A.G. Evans and H.N.G. Wadley (Eds.), MRS Vol. 521, Warrendale, Pennsylvania, USA, 1998, pp. 145-150.

[41] C.E. Wen, Y. Yamada y P.D. Hodgson, Mater. Sci. Eng. C 26 (2006) 1.439-1.444.

[42] H. A. Estupiñán; I. Uribe Pérez; A. Z. Díaz; R. Ulloa y P. Sundaram, Memorias del III Congreso Internacional de Materiales-Simposio Materia 2005-VIII, Congreso Nacional de Corrosión y Protección, Cartagena, Colombia, 2005, pp. 1-8.

[43] S.W. Youn y C.G. Kang, Metall. Materials Trans. B 35 (2004) 769-776.

[44] B. Kriszt, P. Cekan y K. Faure, Proc. 2nd Int. Conf. Cellular metals and metal foaming technology. J. Banhart, M.F. Ashby, N.A. Fleck (Eds.), MIT-Verlag, Bremen, Alemania, 2001, pp. 7782.

[45] S. Asavavisithchai y A.R. Kennedy, J. Colloid Interf. Sci. 297 (2006) 715-723.

[46] M. Arnold, C. Körner y R.F. Singer, Proc. MetFoam 2003 Cellular Metals: Manufacture, properties, applications, J. Banhart, N. A. Fleck, A. Mortensen (Eds.), MIT Press-Verlag, Berlin, Alemania, 2003, pp. 71-76.

[47] E. Proberbio y L. Bonaccorsi, Proc. MetFoam 2003 Cellular Metals: Manufacture, properties, applications, J. Banhart, N. A. Fleck, A. Mortensen (Eds.), MIT Press-Verlag, Berlin, Alemania, 2003, pp. 137-142. 
PROCESOS DE FABRICACIÓN DE METALES CELULARES. PARTE II: VÍA SÓLIDA, DEPOSICIÓN DE METALES, OTROS PROCESOS MANUFACTURING PROCESSES OF CELLULAR METALS. PART II. SOLID ROUTE, METALS DEPOSITION, OTHER PROCESSES

[48] I. Duarte y J. Banhart, Metal Matrix Composites. Euromat 5 (1999) pp. 14-21.

[49] S. Asavavisithchai y A.R. Kennedy, Proc. MetFoam 2003 Cellular Metals: Manufacture, properties, applications, J. Banhart, N. A. Fleck, A. Mortensen (Eds.), MIT Press-Verlag, Berlin, Alemania, 2003, pp. 119-122.

[50] C. Korner, M. Arnold y R.F. Singer, Mater. Sci. Eng. A 396 (2005) 28-40.

[51] S. Esmaeelzadeh, A. Simchi y D. Lehmhus, Mater. Sci. Eng. A 424 (2006) 290-299.

[52] A.R. Kennedy y S. Asavavisitchai, Scr. Mater. 50 (2004) 115-119.

[53] G. Kaptay, Proc. 2nd Int. Conf. Cellular metals and metal foaming technology. J. Banhart, M.F. Ashby, N.A. Fleck (Eds.), MIT-Verlag, Bremen, Alemania, 2001, pp. 117-122.

[54] J. Banhart, J. Baumeister y M. Weber, Proc. European Conf. on Advanced PM Materials (PM'95), European Powder Metallurgy Association, Shrewsbury, Birmingham, Inglaterra, 1995, pp. 201-208.

[55] R. L. Centeno, A.R. Kennedy y J.V. Wood. Proc. 2nd Int. Conf. Cellular metals and metal foaming technology, J. Banhart, M.F. Ashby, N.A. Fleck (Eds.), MIT-Verlag, Bremen, Alemania, 2001, pp. 69-76.

[56] I. Duarte, P. Weigand y J. Banhart, Proc. Int. Conf. Metal foams and porous metal structures, J. Banhart, M. F. Ashby, N.A. Fleck (Eds.), MIT Press-Verlag Bremen, Alemania, 1999, pp. 97 104.

[57] T. Miyoshi, M. Itoh, S. Akiyama, y A. Kitahara, Proc. Int. Conf. Metal foams and porous metal structures, J. Banhart, M. F. Ashby, N.A. Fleck (Eds.), MIT Press-Verlag Bremen, Alemania, 1999, pp. 125-132.

[58] B. Matijaševiç-Lux, A. Rack, A. Haibel, y J. Banhart, Proc. Cellular Metals and Polymers, Fürth, Alemania, 2004, R.F. Singer, C. Körner, V. Altstädt, H. Münstedt, Trans Tech Publications, Zuerich, Switzerland, 2005, pp. 1-5.

[59] B. Matijaševiç-Lux, J. Banhart, S. Fiechter, O. Gorke y N. Wanderka, Acta Mater. 54 (2006) 1.887-1.900.

[60] A.R. Kennedy y V.H. Lopez, Mater. Sci. Eng. A 357 (2003) 258-263.

[61] A.R. Kennedy, V.H. Lopez y S. Asavavisithchai, Proc. MetFoam 2003 Cellular Metals: Manufacture, properties, application, J.Banhart, N. Fleck, A. Mortensen (Eds.), MIT PressVerlag, Berlin, Alemania, 2003, pp. 95-100.

[62] H.-M. Helwig y J. Banhart. Proc., J. Banhart, Proc. MetFoam 2003 Cellular Metals: Manufacture, properties, application, J.Banhart, N. Fleck, A. Mortensen (Eds.), MIT Press-Verlag, Berlin, Alemania, 2003, pp. 165-168.
[63] B. Matijaševiç y J. Banhart, Scr. Mater. 54 (2006) 503-508.

[64] F. Simančik, K. Behulová y L. Bors, Proc. 2nd Int. Conf. Cellular metals and metal foaming technology, J. Banhart, M.F. Ashby, N.A. Fleck (Eds.), MIT-Verlag, Bremen, Alemania, 2001, pp. 89-92.

[65] J. Banhart, Europhys. news 1 (1999) 1-4.

[66] J. Banhart, Proc. Porous Metals and Metal Foaming Technology, H. Nakajima and N. Kanetake (Eds.), The Japan Institute of Metals 2005, Japón, pp. 75-86.

[67] N. Babcsán, D. Leitlmeier y J. Banhart, Colloid Surface A 261 (2005) 123-130.

[68] H. Stanzick, J. Banhart, L. Helfen y T. Baumbach, Proc. Eurofoam2000, P. Zitha, J. Banhart, G. Verbist (Eds.), MIT-Verlag, Bremen, Alemania, 2000, pp. 290-296.

[69] Y. P. Kathuria, J. Mater. Sci. 38 (2003) 2.875 2.881.

[70] A. Irretier y J. Banhart, Acta Mater. 53 (2005) 4.903-4.917.

[71] A. Rack, A. Haibel, A. Bütow, B. Matijašević y J. Banhart. Proc. 16th WCNDT 2004 - World Conference on NDT (Eds.), 2004, Montreal, Canada, pp. 1-9.

[72] F. García-Moreno y N. Kardjilov, Materials world (internet journal) 6, 2005, pp. 1-13. http://www.materialworld.uni-miskolc.hu/tartalom/2005/jan/01_Cellular_Architecture_Gar cia_Kardjilov.pdf (28 octubre 2007)

[73] A. Rack, A. Haibel, B. Matijaševiç y J. Banhart, Proc. MetFoam 2003 Cellular Metals: Manufacture, properties, applications, J. Banhart, N. Fleck, A. Mortensen (Eds.), MIT Press-Verlag, Berlin, Alemania, 2003, pp. 295-300.

[74] T. Baumbach, L. Helfen, K. Schladitz, P. Pernot, H. Stanzick y J. Banhart, Proc. 16th WCNDT 2004-World Conference on NDT (Eds), 2004, Montreal, Canada, pp. 1-8.

[75] E. Cornelis, A. Kottar, A. Sasov y D. Van Dyck, Proc. Int. Conf. Metal foams and porous metal structures, J. Banhart, M. F. Ashby, N.A. Fleck (Eds.), MIT Press-Verlag Bremen, Alemania, 1999, pp. 233-234.

[76] O.B. Olurin, M. Arnold, C. Körner y R.F. Singer, Mater. Sci. Eng. A 328 (2002) 334-343.

[77] Th. Wübben, S. Odenbach y J. Banhart. Proc. Eurofoam2000, P. Zitha, J. Banhart, G. Verbist (Eds.), MIT-Verlag Bremen, Alemania, 2000, pp. 98-103.

[78] J. Banhart, F. Baumgärtner, S. J. Cox, B. Kronberg, D. Langevin, S. Odenbach, D. Weaire y Th. Wübben, Proc. 1st International Symposium on Microgravity Research \& Applications in 
Physical Sciences \& Biotechnology, Sorrento, Italy, 2000, pp. 589-596.

[79] Th. Wübben y S. Odenbach, Colloid Surface A 266 (2005) 207-213.

[80] Th. Wübben, H. Stanzick, J. Banhart, y S. Odenbach, J. Phys.: Condens. Mater. 15 (2003) S427-S433.

[81] Th. Wüebben, S. Odenbach y J. Banhart, Proc. 2nd Int. Conf. Cellular metals and metal foaming technology. J. Banhart, M.F. Ashby, N.A. Fleck (Eds.), MIT-Verlag, Bremen, Alemania, 2001, pp. 83-88.

[82] F. Garcia-Moreno, M. Fromme y J. Banhart, Proc. MetFoam 2003 Cellular Metals: Manufacture, properties, applications, J. Banhart, N. A. Fleck, A. Mortensen (Eds.), MIT Press-Verlag, Berlin, Alemania, 2003, pp. 89-94.

[83] J. Banhart, D. Bellman y H. Clemens, Proc. 2nd Int. Conf. Cellular metals and metal foaming technology. J. Banhart, M.F. Ashby, N.A. Fleck (Eds.), MIT-Verlag, Bremen, Alemania, 2001, pp. 111-116.

[84] J. Banhart, D. Bellmann y H. Clemens, Acta Mater. 49 (2001) 3.409-3.420.

[85] D. Bellman, H. Clemens, B. Matijaševiç, A. Heinemann y J. Banhart, Proc. MetFoam 2003 Cellular Metals: Manufacture, properties, applications, J. Banhart, N. A. Fleck, A. Mortensen (Eds.), MIT Press-Verlag, Berlin, Alemania, 2003, pp. 113-118.

[86] B. Matijašević, S. Fiechter, I. Zizak, O. Görke, N. Wanderka, P. Schubert-Bischoff y J. Banhart, Proc. Cellular Metals and Polymers, Fürth, Alemania, 2004, R.F. Singer, C. Körner, V. Altstädt, H. Münstedt, Trans Tech Publications, Zuerich, Switzerland, 2005, pp. 1-6.

[87] O. Brunke y S. Odenbach, Proc. MetFoam 2003 Cellular Metals: Manufacture, properties, applications, J. Banhart, N. A. Fleck, A. Mortensen (Eds.), MIT Press-Verlag, Berlin, Germany, 2003, pp. 77-82.

[88] B. Matijaševiç, Tesis Doctoral, Technischen Universität Berlin, 2006.

[89] F. Von Zeppelin, M. Hirscher, H. Stanzick y J. Banhart, Compos. Sci. Technol. 63 (2003) 2.293-2.300.

[90] C. Park y S.R. Nutt, Mater. Sci. Eng. A 288 (2000) 111-118.

[91] C. Park y S.R. Nutt, Mater. Sci. Eng. A 297 (2001) 62-68.

[92] A. Makaya y H. Fredriksson, Mater. Sci. Eng. A 413-414 (2005) 533-537.

[93] G. Rausch y T. Hartwig, Proc. 2nd Int. Conf. Cellular metals and metal foaming technology. J. Banhart, M.F. Ashby, N.A. Fleck (Eds.), MITVerlag, Bremen, Alemania, 2001, pp. 203-208.
[94] N. Kanetake y M. Kobashi, Scr. Mater. 54 (2006) 521-525.

[95] J. Baumeister y K. Stöbener, Proc. MetFoam 2003 Cellular Metals: Manufacture, properties, applications, J. Banhart, N. A. Fleck, A. Mortensen (Eds.), MIT Press-Verlag, Berlin, Alemania, 2003, pp. 143-146.

[96] M. Bram, A. Laptev, H.P. Buchkremer y D. Stöver, Proc. Metfoam 2003 Cellular Metals: Manufacture, properties, applications, J. Banhart, N. A. Fleck, A. Mortensen (Eds.), MIT PressVerlag, Berlin, Alemania, 2003, pp 249-252.

[97] L.E. García-Cambronero y C. Ranninger, Proc. MetFoam 2003 Cellular Metals: Manufacture, properties, applications, J. Banhart, N. A. Fleck, A. Mortensen (Eds.), MIT Press-Verlag, Berlin, Alemania, 2003, pp. 175-180.

[98] Y.Y. Zhao y D.X. Sun, Scr. Mater. 44 (2001) 105-110.

[99] K. Nishiyabu, S. Matsuzaki y S. Tanaka, Proc. Cellmet 2005 Cellular Metals for structural and functional applications. G. Stephani and B. Kieback (Eds.), Fraunhofer IRB Verlag, Dresden, Alemania, 2005, pp. 180-187.

[100] D.X. Sun, T. Fung y Y.Y. Zhao, Proc. 2nd Int. Conf. Cellular metals and metal foaming technology. J. Banhart, M.F. Ashby, N.A. Fleck (Eds.), MIT-Verlag, Bremen, Alemania, 2001, pp. 226 230.

[101] D.X. Sun y Y.Y. Zhao, Mater. Lett. 59 (2005) 610.

[102] B. Jiang, N. Zhao, C. Shi, X. Du, J. Li y H. Man, Mater. Lett. 59 (2005) 3.333-3.336.

[103] B. Jiang, N.Q. Zhao, C.S. Shi y J.J. Li, Scr. Mater. 53 (2005) 781-785.

[104] M. Bram, C. Stiller, H.P. Buchkremer, D. Stöver y H. Baur, Proc. Int. Conf. Metal foams and porous metal structures, J. Banhart, M. F. Ashby, N.A. Fleck (Eds.), MIT Press-Verlag Bremen, Alemania, 1999, pp. 197-202.

[105] M. Bram, C. Stiller, H.P. Buchkremer, D. Stöver y H. Baur, Adv. Eng. Mater. 2 (2000) 196-199.

[106] A. Laptev, M. Bram, H. P. Buchkremer y D. Stöver, Powder Metall. 47 (2004) 85-92.

[107] H. I. Bakan, Scr. Mater. 55 (2006) 203-206.

[108] K. Nishiyabu, S. Matsuzaki y S. Tanaka, Proc. MetFoam 2007 Porous Metals and Metallic Foams, L.P. Lefebvre, J. Banhart and D.C. Dunand (Eds.), Destech Publications, Inc. Pensylvania, USA, 2008, pp. 137-140.

[109] Y. Zhao, T. Fung, L. Zhang y F. Zhang, Scr. Mater. 52 (2005) 295-298.

[110] G. Rausch y J. Banhart, Handbook of Cellular Materials - Production, Processing, Applications, H.P. Degischer and B. Kriszt (Eds.), WileyVCH, Weinheim, 2002, pp. 21-28. 
PROCESOS DE FABRICACIÓN DE METALES CELULARES. PARTE II: VÍA SÓLIDA, DEPOSICIÓN DE METALES, OTROS PROCESOS MANUFACTURING PROCESSES OF CELLULAR METALS. PART II. SOLID ROUTE, METALS DEPOSITION, OTHER PROCESSES

[111] O. Andersen y G. Stephani, Handbook of Cellular Materials - Production, Processing, Applications, H.P. Degischer and B. Kriszt (Eds.), Wiley-VCH, Weinheim, 2002, pp. 56-69.

[112] J. Banhart, Prog. Mater. Sci. 46, (2001) 559. 632.

[113] V. N. Antsiferov, A. M. Makarov y V. D. Khramtsov. Adv. Eng. Mater. 7 (2005) 77-92.

[114] Z. Song, S. Kishimoto y N. A. Shinya, Adv. Eng. Mater. 6 (2004) 211-214.

[115] C.E. Wen, M. Mabuchi, Y. Yamada, K. Shimojima, Y. Chino, H. Hosokawa y T Asahina, Proc. MetFoam 2003 Cellular Metals: Manufacture, properties, applications, J. Banhart, N. A. Fleck, A. Mortensen (Eds.), MIT PressVerlag, Berlin, Alemania, 2003, pp. 267-270.

[116] Y-S. Kwon, M-J. Suk, J-S Kim y Ch-G Shin, Proc. MetFoam 2003 Cellular Metals: Manufacture, properties, applications, J. Banhart, N. A. Fleck, A. Mortensen (Eds.), MIT Press-Verlag, Berlin, Alemania, 2003, pp. 257-260.

[117] H. Göhler, P. Löthman, U. Waag, H. Schneidereit y E. Bernhard, Proc. 2nd Int. Conf. Cellular metals and metal foaming technology, J. Banhart, M.F. Ashby, N.A. Fleck (Eds.), MIT-Verlag, Bremen, Alemania, 2001, pp. 391-396.

[118] H. Wadley, Adv. Eng. Mater. 4 (2002) $726-$ 733.

[119] U. Waag, L. Schneider, P. Löthman y G. Stephani, MPR (2000) 29-33.

[120] K. M. Hurysz, J.L Clark, A.R. Nagel, C.U. Hardwicke, K.J. Lee, J.K. Cochran y Jr. T.H. Sanders, Proc. Porous and cellular materials for structural applications, D.S. Schwartz, D.S. Shih, A.G. Evans and H.N.G. Wadley (Eds.), MRS Vol. 521, Warrendale, Pennsylvania, USA, 1998, pp. 191-203.

[121] J.L. Clark, K.M. Hurysz, K.J. Lee, J.K. Cochran y T.H. Sanders, Proc. Int. Conf. Metal foams and porous metal structures, J. Banhart, M. F. Ashby, N.A. Fleck (Eds.), MIT Press-Verlag Bremen, Alemania, 1999, pp. 171-178.

[122] O. Andersen, U. Waag, L. Shneider, G. Stephani y B. Kieback, Adv. Eng. Mater. 2 (2000) 192-195.

[123] D. C. Dunand, Proc. MetFoam 2003 Cellular Metals: Manufacture, properties, applications, J. Banhart, N. A. Fleck, A. Mortensen (Eds.), MIT Press-Verlag, Berlin, Alemania, 2003, pp. 219-230.

[124] D.S. Schwartz, D.S. Shih, R.J. Lederich, R.L. Martin y D.A. Deuser, Proc. Porous and cellular materials for structural applications, D.S. Schwartz, D.S. Shih, A.G. Evans and H.N.G. Wadley (Eds.), MRS Vol. 521, Warrendale, Pennsylvania, USA, 1998, pp. 225-230.
[125] D. M. Elzey y H. N. G. Wadley, Acta Mater. 49 (2001) 849-859.

[126] N.G.D. Murray, C.A. Schuh y D.C. Dunand, Scr. Mater. 49 (2003) 879-883.

[127] D.T. Queheillalt, H.N. Wadley y D.S. Shwrtz, Proc. Porous and cellular materials for structural applications, D.S. Schwartz, D.S. Shih, A.G. Evans and H.N.G. Wadley (Eds.), MRS Vol. 521, Warrendale, Pennsylvania, USA, 1998, pp. 237-242.

[128] D. C. Dunand y J. Teisen, Proc. Porous and cellular materials for structural applications, D.S. Schwartz, D.S. Shih, A.G. Evans and H.N.G. Wadley (Eds.), MRS Vol. 521, Warrendale, Pennsylvania, USA, 1998, pp. 231-236.

[129] N.G.D. Murray y D.C. Dunand, Compos. Sci. Technol. 63 (2003) 2.311-2.316.

[130] M.F. Ashby, A. Evans, N.A. Fleck, L.J. Gibson, J.W. Hutchinson y H.N.G. Wadley, Metal foams: a design guide, Butterworth-Heinemann, Oxford, UK, 2000, pp. 6-23.

[131] K. Kitazono, E. Sato y K. Kuribayashi, Scr. Mater. 50 (2004) 495-498.

[132] K. Kitazono, E. Sato y K. Kuribayashi, Proc. MetFoam 2003 Cellular Metals: Manufacture, properties, applications, J. Banhart, N. A. Fleck, A. Mortensen (Eds.), MIT Press-Verlag, Berlin, Alemania, 2003, pp. 157-160.

[133] U. Mohr, P. F. Scholz, y W. Bleck, Proc. 2nd Int. Conf. Cellular metals and metal foaming technology, J. Banhart, M.F. Ashby, N.A. Fleck (Eds.), MIT-Verlag, Bremen, Alemania, 2001, pp. 209-214.

[134] S. Angel, W. Bleck, P-F. Scholz y T. Fend, Mater. Technol. 75 (2004) 479-484.

[135] Metal foams Mitsubishi. <http://www.mmc. co.jp> (26 enero 2007)

[136] T. Shimizu y K. Matsuzaki, Proc. MetFoam 2003 Cellular Metals: Manufacture, properties, applications, J. Banhart, N. A. Fleck, A. Mortensen (Eds.), MIT Press-Verlag, Berlin, Alemania, 2003, pp. 253-256.

[137] J.S. Kim, J.H. Kang, S.B. Kang, K.S. Yoon y Y.S. Kwon, Proc. MetFoam 2003 Cellular Metals: Manufacture, properties, applications, J. Banhart, N. A. Fleck, A. Mortensen (Eds.), MIT PressVerlag, Berlin, Alemania, 2003, pp. 231-236.

[138] M. Kobashi y N. Kanetake, Proc. 2nd Int. Conf. Cellular metals and metal foaming technology. J. Banhart, M.F. Ashby, N.A. Fleck (Eds.), MIT-Verlag, Bremen, Alemania, 2001, pp. 221-225.

[139] N. Kanetake y M. Kobashi, Proc. Cellmet 2005 Cellular Metals for structural and functional applications. G. Stephani and B. Kieback (Eds.), Fraunhofer IRB Verlag, Dresden, Alemania, 2005. pp. 131-136. 
[140] M. Kobashi y N. Kanetake, Proc. MetFoam 2003 Cellular Metals: Manufacture, properties, applications, J. Banhart, N. A. Fleck, A. Mortensen (Eds.), MIT Press-Verlag, Berlin, Alemania, 2003, pp. 237-242.

[141] A. Verdooren, H. M. Chan, J. L. Grenestedt, M. P. Harmer y H. S. Caram., J. Mater. Sci. 40 (2005) 4.333-4.339.

[142] A. Verdooren, H. M. Chan, J. L. Grenestedt, M. P. Harmer, H. S. Caram, Adv. Eng. Mater. 6 (2004) 397-399.

[143] A. Verdooren, H. M. Chan, J. L. Grenestedt, M. P. Harmer, H. S. Caram, Proc. MetFoam 2003 Cellular Metals: Manufacture, properties, applications, J. Banhart, N. A. Fleck, A. Mortensen (Eds.), MIT Press-Verlag, Berlin, Alemania, 2003, pp. 243-248.
[144] H. Verduyn, J. Leinjerhorts y A. TriesScheijnNooteboom, Proc. Cellmet 2005 Cellular Metals for structural and functional applications. G. Stephani and B. Kieback (Eds.), Fraunhofer IRB Verlag, Dresden, Alemania, 2005. pp. 91-94.

[145] V. Paserin, S. Marcuson, J. Shu y D.S. Wilkinson, Proc. MetFoam 2003 Cellular Metals: Manufacture, properties, applications, J. Banhart, N. A. Fleck, A. Mortensen (Eds.), MIT Press-Verlag, Berlin, Alemania, 2003, pp. 31-38.

[146] Inco Ltd., Canada, Product data sheet of "Incofoam", 1998, http://www.inco.com (18 julio 2005).

[147] H. Choe y D. C. Dunand, Acta Mater. 52 (2004) 1.283-1.295. 